\title{
EQUATIONS WITH CONSTANT COEFFICIENTS INVARIANT UNDER A GROUP OF LINEAR TRANSFORMATIONS
}

\author{
BY \\ ANDRÉ CEREZO
}

\begin{abstract}
If $P$ is a linear differential operator on $R^{n}$ with constant coefficients, which is invariant under a group $G$ of linear transformations, it is not true in general that the equation $P u=f$ always has a $G$-invariant solution $u$ for a $G$-invariant $f$. We elucidate here the particular case of a "big" group $G$, and we count the invariant solutions when they exist (see Corollary 28 and Theorems 32, 33). The case, of special interest, of the wave equation and the Lorentz group is covered (Corollary 27). The theory of hyperfunctions provides the frame for the work.
\end{abstract}

Introduction. In his note [6], M. Rais proves that every linear differential operator $\boldsymbol{P}$ on $\mathbf{R}^{\boldsymbol{n}}$ with constant coefficients has a tempered fundamental solution invariant under the subgroup of $\mathrm{GL}(n, \mathbf{R})$ under which $P$ is invariant: it is a consequence of a theorem of M. F. Atiyah [1]. But it is not true in general that $P$ has an invariant solution of any equation whose second side is invariant by the same group, and $M$. Rais presents a counterexample where $P$ has degree one.

We study here this question. As, in general, when a group acts on a manifold, some orbits are "singular" (the quotient is not a manifold), one is led naturally to solve an invariant equation first on the open set of the regular orbits, and then to try to extend the solutions: in order to eliminate any artificial obstruction there might be to such an extension, we will work within the frame of the theory of hyperfunctions. To this theory, first developed in [8], one will find a short and excellent introduction in [3].

We have to make precise here what we mean by "invariant". This is clear for a function, and therefore for a hyperfunction; for a distribution, there are two possible definitions: the first extends the definition for functions; the second is the one used in $[6, \S 1.4]$, for which the Dirac measure is invariant under all transformations of the linear group, and for which one has the 
TheOREm (AtIYAh-RAIS). Every linear differential operator $\boldsymbol{P}$ on $\mathbf{R}^{\boldsymbol{n}}$ with constant coefficients has a tempered fundamental solution which is invariant under the subgroup of $\mathrm{GL}(n, \mathbf{R})$ which leaves $P$ invariant.

It should be remarked that the Dirac measure $\delta$ is not invariant under $\mathbf{G L}(n, \mathbf{R})$ in the first sense: it is well known for instance that, on $\mathbf{R}, \delta$ is homogeneous of degree -1 . However, the two definitions coincide if we consider only the subgroup of $\operatorname{GL}(n, \mathrm{R})$ (noted here $G_{n}$ ) of the linear transformations of jacobian \pm 1 . As this restriction is of no consequence in the most interesting case we look at here (that is when the operator is invariant under a general orthogonal group), we choose to stick to it from now on.

The problem is thus the following: $P=P\left(\partial_{x}\right)$ is a linear differential operator on $\mathbf{R}^{n}$ with constant coefficients, $G$ is the subgroup of $G_{n}$ under which $P$ is invariant (that is to say, for instance, that the distribution $P \delta$ is invariant); $G$ is obviously a closed (algebraic) subgroup of $\operatorname{GL}(n, \mathbf{R})$; let $G_{0}$ be its connected component of the identity, and $f \in B\left(\mathbf{R}^{n}\right)$ a hyperfunction on $\mathbf{R}^{n}$ invariant under $G$ (resp. $G_{0}$ ). Are there solutions $u \in B\left(\mathbf{R}^{n}\right)$, invariant under $G$ (resp. $G_{0}$ ), of the equation $P u=f$, and how many?

$G$ is obviously the intersection of the groups which leave invariant the real part or the imaginary part of each of the homogeneous parts of $P$. In order to find a big enough group $G$, we will thus restrict our search here to the case where $\boldsymbol{P}$ is a polynomial (with complex coefficients) in an operator $\Pi$, homogeneous and with real coefficients, of degree 1 or 2 . The principal difficulty lies then in the extension at the origin of a hyperfunction on $\mathbf{R}^{n}-\{0\}$ invariant under the orthogonal group of some quadratic form.

We obtain a complete answer to the questions above in both cases (invariance under $G$ or $G_{0}$-see Theorems 28 and 32-33), and our results apply in particular to the case where $\Pi$ is the wave operator and the group of invariance is the Lorentz group.

$\S 1$ studies the hyperfunctions on $\mathbf{R}^{n}-\{0\}$ and on $\mathbf{R}^{n}$ which are invariant under a noncompact orthogonal group, assuming $n \geqslant 3$. We look in detail at the case of $\mathbf{R}^{\mathbf{2}}$ in $\S 2$, as it presents a special difficulty, of a topological nature. In $\S 3$ we state and prove the results on invariant equations which are the aim of this work.

Apart from [4] , [6], and older works like [7], particular cases of this problem have been treated in [11]-[17].

Finally I would like to thank here F. Rouviere, who simplified considerably the original proof of Theorem 10.

1. Hyperfunctions invariant under a noncompact orthogonal group. We begin this section with a few generalities on invariance of a hyperfunction under 
a group. We first recall that, if $M$ is an $n$-dimensional real analytic manifold and $X$ a complexification of $M$, the collection of the spaces

$$
H^{n}\left(X-(M-U) \bmod X-M, O_{X}\right)
$$

for all open subsets $U$ of $M$, together with the canonical restrictions, constitutes the flabby sheaf $B$ of the hyperfunctions on $M$, and that it is in a natural way a sheaf of $D$-modules, where $D$ is the sheaf of linear differential operators with analy tic coefficients on $M$.

Representing $H^{n}\left(X-(M-U) \bmod X-M, O_{X}\right)=B(U)$ by the corresponding group of Čech cohomology associated to a relative covering of $(X, X-M)$ by Stein open sets, one obtains a representation of any hyperfunction $f(x)$ on an open subset of $M$ by relative Cech $n$-cocycles $\varphi(z)$, which are families of functions holomorphic in certain subsets of $X-M$ (two such cocycles represent the same hyperfunction if their difference is a coboundary).

If $G$ is a Lie group of diffeomorphisms of $M$, the transformations $g \in G$, being analytic, act also on the relative cocycles. A hyperfunction $f(x)$ on $M$ represented by a cocycle $\varphi(z)$ is invariant under $G$ if and only if

$$
\forall g \in G \quad f(g x)=f(x),
$$

that is

$$
\forall g \in G \quad \varphi(g z)=\varphi(z)+\psi(g, z)
$$

where $\psi(g, z)$ is a relative $n$-coboundary, depending analytically on $g \in G$. But in general there is no relative cocycle $\varphi$ representing $f$ and itself invariant under $G$ (i.e. such that $\varphi(g z)=\varphi(z)$ for every $g \in G$ ), as the following example shows:

On $\mathbf{R}$, consider the group $G$ of homotheties of strictly positive ratio. The relative 1-cocycles can be realized as holomorphic functions in $\mathbf{C}-\mathbf{R}$, and these are $G$-invariant if and only if they are locally constant. The $G$-invariant hyperfunctions obtained in this way are thus the constants. But the Heaviside function

$$
H(x)=[\ln (-z)]_{z=x}
$$

where $\ln$ is the principal determination of the logarithm (holomorphic except on the negative real half-line), is of course $G$-invariant:

$$
\ln (-a z)=\ln (-z)+\ln a \quad(a>0) .
$$

Let now $G_{0}$ be a connected Lie group of diffeomorphisms of $M$, and $\left(X_{i}\right)_{i=1, \cdots, r}$ a basis of the Lie algebra of $G_{0}$. We identify $X_{i}$ with the vector field on $M$, right-invariant under $G_{0}$, generated by $X_{i}$ : if $f$ is a (hyper-)func- 
tion on an open subset of $M$,

$$
X_{i} f(x)=\left.\frac{d}{d t}\right|_{t=0} f\left(\exp t X_{i} \cdot x\right) .
$$

Lemma 1. Let $U$ be a $G_{0}$-invariant open subset of $M$, and $f$ a hyperfunction on $U . f$ is $G_{0}$-invariant if and only if $X_{i} f=0 \quad(i=1, \cdots, r)$.

Proof. Let $g f$ be the image of $f$ under $g \in G_{0}: g f(x)=f(g x)$, and $\varphi(z)$ a relative $n$-cocycle representing $f$. If $f$ is $G_{0}$-invariant, for any $g \in G_{0}$,

$$
\varphi(g z)-\varphi(z)=\psi(g, z)
$$

is a relative $n$-coboundary depending analytically on $g$, and the hyperfunction $X_{i} f$ is represented by the coboundary

$$
X_{i} \varphi(z)=\left.\frac{d}{d t}\right|_{t=0} \varphi\left(\exp t X_{i} \cdot z\right)=\left.\frac{d}{d t}\right|_{t=0} \psi\left(\exp t X_{i} \cdot z\right),
$$

thus it is zero. If reciprocally $X_{i} f=0(i=1, \cdots, r)$, we have

$$
\left.\frac{d}{d t}\right|_{t=0} \varphi\left(\exp t X_{i} \cdot z\right)=\chi(t, z)
$$

where $\chi$ is a relative $n$-coboundary depending analytically on $t \in \mathbf{R}$; thus

$$
\varphi\left(\exp t X_{i} \cdot z\right)=\varphi(z)+\int_{0}^{t} \chi(u, z) d u
$$

where the integral is still a coboundary, and this implies $f\left(\exp t X_{i} \cdot x\right)=f(x)$ and finally $g f=f$ for any $g \in G_{0}$, since $G_{0}$ is connected. Q.E.D.

Lemma 1 shows that the hyperfunctions on an open set $U$ of $M$ which are $G_{0}$-invariant form the kernel of the application

$$
\left(X_{i}\right): B(U) \rightarrow B^{r}(U) .
$$

One can thus talk of the sheaf $B^{G_{0}}$ of the locally $G_{0}$-invariant hyperfunctions: it is the kernel of the sheaf morphism

$$
\left(X_{i}\right): B \rightarrow B^{r} .
$$

In particular, if $G$ is a connected one parameter Lie group, and $X$ its infinitesimal generator, and if $U$ is open in $M$ and $G_{0}$-invariant, we denote $\pi: B^{G_{0}}(M) \rightarrow B^{G_{0}}(U)$ the canonical restriction, $F=M-U$, and $B_{F}$ the sheaf of hyperfunctions supported in $F$.

LEMmA 2. If $X: B(M) \rightarrow B(M)$ is onto, there is a canonical isomorphism between $B^{G_{0}}(U) / \pi B^{G_{0}}(M)$ and $B_{F}(M) / X B_{F}(M)$.

Proof. It is given by the following exact commutative diagram: 


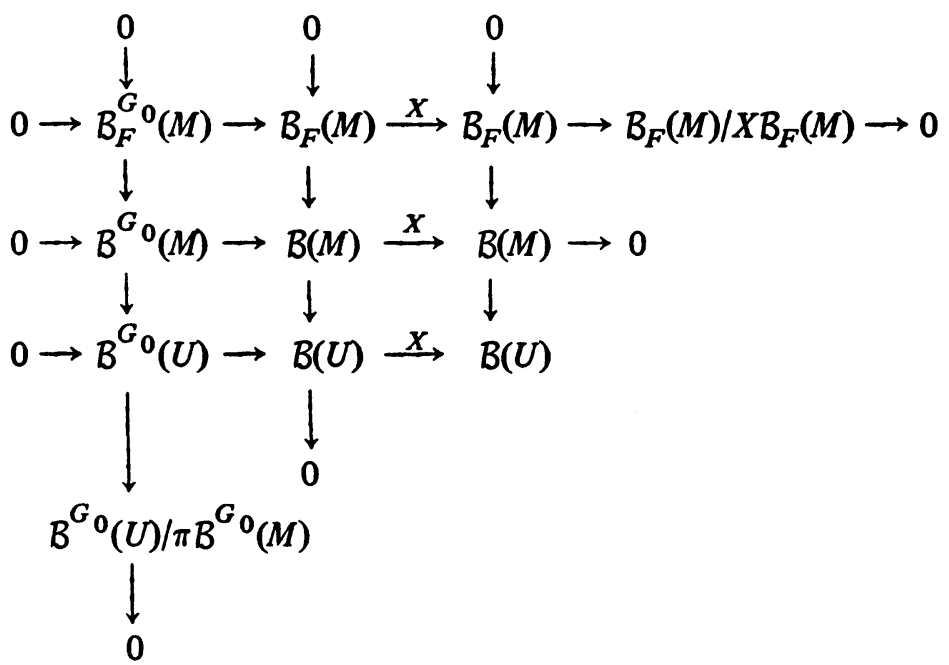

REMARK. It is known that every hyperfunction on $U$ can be extended to a hyperfunction on $M$; one could wonder whether any $G_{0}$-invariant hyperfunction on $U$ admits at least one $G_{0}$-invariant extension. This is not true in general, and the preceding lemma, which enlightens the situation, will be used to prove it (Propositions 12, 13).

REMARK 3. Lemma 2, as its proof shows, is actually much more general: if $F$ is a sheaf on $M, P: F \rightarrow F$ a sheaf morphism, $F^{P}$ its kernel sheaf, $U$ open in $M, F=M-U, F_{F}$ the sheaf of sections supported in $F$ :

If the restriction of $F$ from $M$ to $U$ is onto, and if $P$ is onto on the global sections, there is a canonical isomorphism between $F^{P}(U) / F^{P}(M)$ and $F_{F}(M) / P F_{F}(M)$.

It is given by the same diagram, with an obvious change of notations. This obvious remark will be used in the proof of Lemma 29.

From now on in this section we study the $G$-invariant hyperfunctions on $\mathbf{R}^{p+q}$, where $G=O(p, q)$ is the group of matrices which leave invariant the quadratic form

$$
Q(x)=x_{1}^{2}+\cdots+x_{p}^{2}-x_{p+1}^{2}-\cdots-x_{p+q}^{2}
$$

and the hyperfunctions invariant only under its connected component of the identity $G_{0}$.

REMARK. $G$ is self-transposed, and it is also the subgroup of $\mathrm{GL}(p+q, \mathbf{R})$ which leaves invariant the operator

$$
\Pi=\partial_{x_{1}}^{2}+\cdots+\partial_{x_{p}}^{2}-\partial_{x_{p+1}}^{2}-\cdots-\partial_{x_{p+q}}^{2}
$$

and thus any polynomial of $\Pi$. 
We assume $p q>1$; that is to say, we assume that the groups $G$ and $G_{0}$ are not compact $(p q=0)$, and we leave aside the particular case $p=q=1$, which will be treated in $\S 2$.

The Lie algebra of $G$ and $G_{0}$ is the algebra of matrices

$$
\left(\begin{array}{cc}
A & C \\
{ }^{t} C & B
\end{array}\right)
$$

where $A$ and $B$ are antisymmetric, $p \times p$ and $q \times q$ respectively, and $C$ is $p \times q$. A basis is given by the matrices of this kind which have only one nonzero entry, equal to 1 , above the diagonal; so we get:

(1) $p(p-1) / 2+q(q-1) / 2$ vectors $X_{i j}(1 \leqslant i<j \leqslant p$ or $p+1 \leqslant$ $i<j \leqslant p+q)$ generating $\left(\begin{array}{ll}A & 0 \\ 0 & B\end{array}\right)$. $\left(\begin{array}{cc}0 & C \\ t_{C} & 0\end{array}\right)$.

(2) $p q$ vectors $Y_{i j}(1 \leqslant i \leqslant p$ and $p+1 \leqslant j \leqslant p+q)$ generating

The right $G$-invariant differential operators generated by these vectors are, respectively:

(1) $X_{i j}=x_{i} \partial_{j}-x_{j} \partial_{i}(1 \leqslant i<j \leqslant p$ or $p+1 \leqslant i<j \leqslant p+q)$,

(2) $Y_{i j}=x_{i} \partial_{j}+x_{j} \partial_{i}(1 \leqslant i \leqslant p$ and $p+1 \leqslant j \leqslant p+q)$.

We write $\partial_{i}=\partial_{x_{i}}(1 \leqslant i \leqslant p+q)$, and we will also use the notations $X_{i j}$, $Y_{i j}$ with $i>j$ : the $X_{i j}$ are then antisymmetric, and the $Y_{i j}$ symmetric. The vectors $X_{i j}$ generate a compact subgroup $G_{1}$ of $G_{0}$, isomorphic to $\mathrm{SO}(p) \times$ $\mathrm{SO}(q)$.

Intuitively a $G$-invariant hyperfunction on $\mathrm{R}^{p+q}$ is a hyperfunction which "depends only on $Q(x)$ "; this is what is expressed in Proposition 5.

Lemma 4. Let $U$ be open in $\mathbf{R}^{r+s}$, and $V \subset \mathbf{R}^{s}$ be its natural (open) projection. Assume that the fibres of $U$ above $V$ are connected. A hyperfunction $f\left(x_{1}, \cdots, x_{r+s}\right) \in B(U)$ satisfies $\partial f / \partial x_{j}=0(j=1, \cdots, r)$ if and only if $f$ is the inverse image under the natural projection of some hyperfunction on $V$.

We do not give any proof here of this well-known lemma.

Let $Q$ denote the analytic map from $\mathbf{R}^{p+q}-\{0\}$ into $\mathbf{R}$ :

$$
Q(x)=Q\left(x_{1}, \cdots, x_{p+q}\right)=x_{1}^{2}+\cdots+x_{p}^{2}-x_{p+1}^{2}-\cdots-x_{p+q}^{2} .
$$

Proposition 5. A hyperfunction on $\mathbf{R}^{p+q}-\{0\}$ is G-invariant if and only if it is the inverse image under $Q$ of some hyperfunction on $\mathbf{R}$.

Proof. As $Q$ is of maximal rank at any point of its domain, every hyperfunction on $\mathbf{R}$ has an inverse image under $Q$ (this is a consequence of $[9$, Theorem 2.2.6, p. 292]). Let $f \in B\left(\mathbf{R}^{p+q}-\{0\}\right)$ be $G$-invariant. Choose two 
integers $k$ and $l$, such that $1 \leqslant k \leqslant p$ and $p+1 \leqslant l \leqslant p+q$, and let $E_{k l}^{+}$ (resp. $E_{k l}^{-}$) be the open half-space of $\mathrm{R}^{p+q}$ defined by $x_{k}>x_{l}$ (resp. $x_{k}<x_{l}$ ). The analytic map

$$
\begin{aligned}
\theta_{k l}^{-1}:\left(x_{1},\right. & \left.\cdots, x_{p+q}\right) \\
& \mapsto\left(x_{k}-x_{l}=u, Q(x)=v, x_{1}, \cdots \hat{x}_{k}, \cdots, \hat{x}_{l}, \cdots, x_{p+q}\right)
\end{aligned}
$$

is a diffeomorphism of $E_{k l}^{ \pm}$onto $\mathbf{R}^{ \pm} \times \mathbf{R}^{p+q-1}$.

Since $f$ is $G_{0}$-invariant, it must satisfy, according to Lemma 1:

$$
X_{i j} f=Y_{i j} f=0 \text { in } E_{k l}^{ \pm}
$$

and by the coordinate change $\theta_{k l}$, the operators

$$
\begin{array}{ll}
X_{i j} & (1 \leqslant i<j \leqslant p \text { or } p+1 \leqslant i<j \leqslant p+q), \\
Y_{i j} & (1 \leqslant i \leqslant p \text { and } p+1 \leqslant j \leqslant p+q)
\end{array}
$$

transform into

$$
\begin{aligned}
X_{i j}^{\prime} & =x_{i} \partial_{j}-x_{j} \partial_{i} \quad(i \text { and } j \text { distinct from } k \text { and } l), \\
X_{k j}^{\prime} & =x_{k} \partial_{j}-x_{j} \partial_{u}, \\
X_{i l}^{\prime} & =-x_{l} \partial_{i}-x_{i} \partial_{u}, \\
Y_{i j}^{\prime} & =x_{i} \partial_{j}+x_{j} \partial_{i} \quad(i \neq k \text { and } j \neq l), \\
Y_{k j}^{\prime} & =x_{k} \partial_{j}+x_{j} \partial_{u}, \\
Y_{i l}^{\prime} & =x_{l} \partial_{i}-x_{i} \partial_{u}, \\
Y_{k l}^{\prime} & =\left(x_{k}-x_{l} \partial_{u}=u \partial_{u} .\right.
\end{aligned}
$$

The system of equations $X_{i j}^{\prime} \theta_{k l} f=Y_{i j}^{\prime} \theta_{k l} f=0$ clearly implies $\partial_{u} \theta_{k l} f=0$ and $\partial_{i} \theta_{k l} f=0$ for $i \neq k, l$ (and reciprocally). Applying Lemma 4, we see that $\left.f\right|_{E_{k l}^{ \pm}}$is the inverse image under $Q$ of some hyperfunction $T_{k l}^{ \pm}$on $\mathbf{R}$.

As $f$ is invariant under the simultaneous change of $x_{k}$ into $-x_{k}$ and $x_{l}$ into $-x_{l}$, one can assume $T_{k l}^{+}=T_{k l}^{-}=T_{k l}$; as $f$ is invariant under the simultaneous change of $x_{l}$ into $x_{l^{\prime}}$ and $x_{l^{\prime}}$ into $-x_{l}$ on one hand, and of $x_{k}$ into $x_{k^{\prime}}$ and $x_{k^{\prime}}$ into $-x_{k}$ on the other hand, one can assume that all hyperfunctions $T_{k l}$ are equal to some hyperfunction $T$ on $\mathbf{R}$.

If reciprocally $f$ is the inverse image under $Q$ of some $T \in B(R)$, Lemma 4 implies

$$
X_{i j}^{\prime} \theta_{k l} f=Y_{i j}^{\prime} \theta_{k l} f=0,
$$

thus $X_{i j} f=Y_{i j} f=0$ in $E_{k l}^{ \pm}$for any $k, l$. There follows that $f$ is $G_{0}$-invariant, 
and as it obviously satisfies the symmetry conditions above, and is furthermore invariant under the change of $x_{i}$ into $-x_{i}(1 \leqslant i \leqslant p+q)$, it is $G$-invariant. Q.E.D.

REMARK 6. If $p$ and $q$ are both strictly larger than one, and if $f$ is $G_{0}$-invariant, define the $T_{k l}$ as above. As $f$ is invariant under the simultaneous change of $x_{k}$ into $-x_{k^{\prime}}, x_{k^{\prime}}$ into $-x_{k^{\prime}}, x_{l}$ into $-x_{l}, x_{l^{\prime}}$ into $-x_{l^{\prime}}$, one can take $T_{k l}^{+}=T_{k l}^{-}=T_{k l}$, and the rest of the proof above remains true, so that: If $p>1$ and $q>1$, every $G_{0}$-invariant hyperfunction on $\mathbf{R}^{p+q}-\{0\}$ is $G$ invariant.

If on another hand $q=1$ (the situation is evidently symmetric in $p$ and q), that is in the case of a Lorentz group, one can, as in the beginning of the proof of Proposition 5, find hyperfunctions on $\mathbf{R}, T_{k l}$, whose inverse images under $Q$ are the $\left.f\right|_{E k l}$, as soon as $f$ is $G_{0}$-invariant. Moreover $f$ is then invariant under the rotations transforming $x_{k}$ into $x_{k^{\prime}}$ and $x_{k^{\prime}}$ into $-x_{k}$, and one can thus assume

$$
T_{k l}^{+}=T_{k^{\prime} l}^{+}=T^{+} \quad \text { and } \quad T_{k l}^{-}=T_{k^{\prime} l}^{-}=T^{-} .
$$

We call $\mathbf{R}^{\prime}$ the non-Hausdorff manifold obtained by gluing together the open negative half-lines of two copies of $\mathbf{R}$, say $\mathbf{R}_{\mathbf{1}}$ and $\mathbf{R}_{\mathbf{2}}$. A hyperfunction on $\mathbf{R}^{\prime}$ will be a pair of hyperfunctions on $R$, say $T_{1}$ and $T_{2}$, whose restrictions to the open negative half-line coincide. Finally let us define an analytic map $\boldsymbol{Q}^{\prime}$ from $\mathbf{R}^{p+1}-\{0\}$ into $\mathbf{R}^{\prime}$ as the pair of analytic maps $Q_{j}: U_{j} \rightarrow \mathbf{R}_{j}(j=$ $1,2)$ defined by $Q_{j}(x)=Q(x)(j=1,2)$,

$$
\begin{aligned}
& U_{1}=\left\{\left(x_{1}, \cdots, x_{p+1}\right) \in \mathbf{R}^{p+1}-\{0\} \mid Q(x)<0 \text { or } x_{p+1}>0\right\}, \\
& U_{2}=\left\{\left(x_{1}, \cdots, x_{p+1}\right) \in \mathbf{R}^{p+1}-\{0\} \mid Q(x)<0 \text { or } x_{p+1}<0\right\} .
\end{aligned}
$$

The inverse image under $Q^{\prime}$ of a hyperfunction on $\mathbf{R}^{\prime}$ is a hyperfunction on $\mathbf{R}^{p+1}-\{0\}$ defined in the obvious way.

PRoposition 7. If $p>1$, the $G_{0}$-invariant hyperfunctions on $\mathbf{R}^{p+1}-\{0\}$ are the inverse images under $Q^{\prime}$ of the hyperfunctions on $\mathbf{R}^{\prime}$.

Proof. It is analogous to the proof of Proposition 5.

REMARK. Clearly, if $T$ is a distribution on $\mathbf{R}$ or $\mathbf{R}^{\prime}$, its inverse image under $Q$ or $Q^{\prime}$ is a distribution on $\mathbf{R}^{p+q}-\{0\}$, and reciprocally, since it is enough to check it locally. Thus we find again Theorem 2 of [4]:

Corollary. Propositions 5 and 7 (and Remark 6) are still true if one replaces everywhere "hyperfunction" by "distribution". 
Let $B_{0}$ be the space of hyperfunctions on $\mathbf{R}^{p+q}$ supported at the origin. We recall that the elements of $B_{0}$ are the series of derivatives of the Dirac measure $\delta, \Sigma a_{\alpha} \delta^{(\alpha)}(\alpha$ is a $(p+q)$-index), which are convergent in $B$, that is such that

$$
\limsup _{|\alpha| \rightarrow \infty}\left(\left|a_{\alpha}\right| \alpha !\right)^{1 /|\alpha|}=0 .
$$

PROPOSITION 8. The $G_{0}$-invariant hyperfunctions supported at the origin have the form $\Sigma_{\alpha \in \mathrm{N}} a_{\alpha} \Pi^{\alpha} \delta$ where $\Pi=\partial_{1}^{2}+\cdots+\partial_{p}^{2}-\partial_{p+1}^{2}-\cdots-\partial_{p+q}^{2}$, and

$$
\limsup _{\alpha \rightarrow \infty}\left(\left|a_{\alpha}\right|(2 \alpha) !\right)^{1 / \alpha}=0
$$

All these are G-invariant.

Proof (CF. P. D. Methée [4, Theorem 1]). We can assume for instance $p>1$. If $\Sigma a_{\alpha} \delta^{(\alpha)}$ is $G_{0}$-invariant, the entire function $F(z)=\Sigma a_{\alpha} z^{\alpha}$ is invariant under the transpose of $G_{0}$, which is no other than $G_{0}$. Since $p>1, G_{0}$ contains a rotation bringing $\left(x_{1}, 0, \cdots, 0\right)$ onto $\left(-x_{1}, 0, \cdots, 0\right)$. Thus

$$
F\left(x_{1}, 0, \cdots, 0\right)=F\left(-x_{1}, 0, \cdots, 0\right)=G\left(x_{1}^{2}\right)
$$

where $G$ is an entire function. The entire function

$$
F\left(x_{1}, \cdots, x_{p+q}\right)-G(Q(x))
$$

is then $G_{0}$-invariant, and vanishes on the first axis; since $G_{0}$ contains transformations bringing any point in the cone $\{x \mid Q(x)>0\}$ onto a point on the first axis, it vanishes identically.

We still have to write that the series $f=\Sigma_{\alpha \in N} a_{\alpha} \Pi^{\alpha} \delta$ is convergent in $B_{0}$. Since

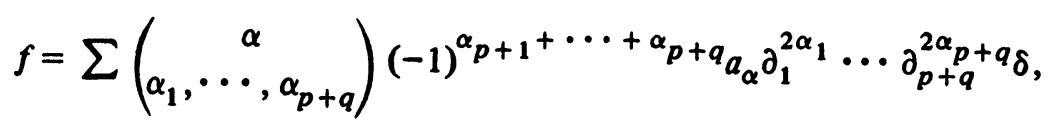

condition (C) gives

$$
\text { (*) } \limsup _{\substack{|\alpha| \rightarrow \infty \\ \alpha_{1}+\cdots+\alpha_{p+q}=\alpha}}\left[\left|a_{\alpha}\right| \frac{\alpha !}{\alpha_{1} ! \cdots \alpha_{p+q} !}\left(2 \alpha_{1}\right) ! \cdots\left(2 \alpha_{p+q}\right) !\right]^{1 / 2|\alpha|}=0 \text {. }
$$

Since

$$
\frac{\left(\alpha_{1}+\cdots+\alpha_{p+q}\right) !}{\alpha_{1} ! \cdots \alpha_{p+q} !} \leqslant \frac{\left(2 \alpha_{1}+\cdots+2 \alpha_{p+q}\right) !}{\left(2 \alpha_{1}\right) ! \cdots\left(2 \alpha_{p+q}\right) !}
$$


this condition will be satisfied as soon as

$$
\limsup _{\substack{\alpha \rightarrow \infty \\ \alpha_{1}+\cdots+\alpha_{p+q}=\alpha}}\left(\left|a_{\alpha}\right|\left(2 \alpha_{1}+\cdots+2 \alpha_{p+q}\right) !\right)^{1 / 2|\alpha|}=0,
$$

which is to say $\left(C^{\prime}\right)$. But $\left(C^{\prime}\right)$ is also necessary, as $(*)$ implies it as one takes $\alpha_{1}=\alpha$ and $\alpha_{2}=\cdots=\alpha_{p+q}=0$. Finally the last assertion is evident, since $\Pi$ and $\delta$ are $G$-invariant. Q.E.D.

REMARK 9. This proposition still holds if $p$ or $q$ is zero, that is if $\Pi$ is elliptic, and the proof is the same.

The end of the chapter is devoted to the proof of the following result:

THEOREM 10. Under the hypothesis $p q \neq 1$, every $G_{0}$-invariant hyperfunction on $\mathbf{R}^{p+q}-\{0\}$ can be extended to a $G_{0}$-invariant hyperfunction on $\mathbf{R}^{p+q}$.

Proof. The case $p q=0$ is trivial, since $G_{0}$ is compact: it is enough then to take an arbitrary extension at the origin, and to integrate its transforms under $G_{0}$ with respect to the Haar measure of $G_{0}$. Thus we assume $p q>1$, and for instance $p>1$.

Let $f$ be a $G_{0}$-invariant hyperfunction on $\mathbf{R}^{p+q}-\{0\}$, and $\widetilde{f}$ an arbitrary extension of $f$ on $\mathbf{R}^{p+q}$. If $\left(X_{i j}\right)(1 \leqslant i<j \leqslant p$ or $p+1 \leqslant i<j \leqslant$ $p+q)$ and $\left(Y_{i j}\right)(1 \leqslant i \leqslant p$ and $p+1 \leqslant j \leqslant p+q)$ are the infinitesimal generators of $G_{0}$ defined above, the $X_{i j}$ generate a compact subgroup $G_{1}$ of $G_{0}$, of normalized Haar measure $d g_{1}$; replacing possibly $\widetilde{f}(x)$ by $\int_{G_{1}} \widetilde{f}\left(g_{1} x\right) d g_{1}$, we can assume that $\widetilde{f}$ is $G_{1}$-invariant, thus that

$$
X_{i j} \tilde{f}=0 \quad(1 \leqslant i<j \leqslant p \text { or } p+1 \leqslant i<j \leqslant p+q) .
$$

As $f$ is $G_{0}$-invariant, the $Y_{i j} \widetilde{f}$ are hyperfunctions supported at the origin. From the commutation relations

$$
\left[X_{i j}, Y_{j k}\right]=Y_{i k} \text { and }\left[Y_{i j}, Y_{k j}\right]=X_{i k}
$$

(the $Y_{i j}$ are symmetric, and the $X_{i j}$ antisymmetric), we deduce, taking (1) into account

$$
Y_{i k} \tilde{f}=X_{i j} Y_{j k} \tilde{f} \text { and } Y_{i k} Y_{j k} \tilde{f}=Y_{j k} Y_{i k} \tilde{f} \text {. }
$$

Choose three integers $i, i^{\prime}, j$ such that $1 \leqslant i \leqslant p, \quad 1 \leqslant i^{\prime} \leqslant p, i \neq i^{\prime}, p+1 \leqslant$ $j \leqslant p+q$. Clearly $\partial_{i^{\prime}} Y_{i j}-\partial_{i} Y_{i^{\prime} j}=\partial_{j} X_{i i^{\prime}}$ so that (1) implies

$$
\partial_{i^{\prime}}\left(Y_{i j} \widetilde{f}\right)=\partial_{i}\left(Y_{i^{\prime} j} \widetilde{f}\right) .
$$


But by developing the hyperfunctions $Y_{i j} \tilde{f}$ and $Y_{i^{\prime} j} \tilde{f}$ as series of derivatives of the Dirac measure, one sees easily that the last equality means that there exists a hyperfunction $g_{j} \in B_{0}$ such that $Y_{i j} \tilde{f}=\partial_{i} g_{j}$ and $Y_{i^{\prime} j} \tilde{f}=\partial_{i^{\prime}} g_{j}$. Of course leaving $j$ fixed and changing the first indices, one proves in the same way that

$$
Y_{i j} \tilde{f}=\partial_{i} g_{j} \quad \text { for every } i=1, \cdots, p .
$$

We now show that $g_{j}$ is invariant under the subgroup of $G_{0}$ generated by the $X_{i i^{\prime}}\left(1 \leqslant i<i^{\prime} \leqslant p\right)$ : the rotation $\rho$ of angle $\theta$ on the variables $x_{i}$ and $x_{i^{\prime}}$ transforms $\partial_{i}$ into $\cos \theta \partial_{i}+\sin \theta \partial_{i^{\prime}}$, and $Y_{i j}=x_{i} \partial_{j}+x_{j} \partial_{i}$ into $\cos \theta\left(x_{i} \partial_{j}+x_{j} \partial_{i}\right)+\sin \theta\left(x_{i^{\prime}} \partial_{j}+x_{j} \partial_{i^{\prime}}\right)=\cos \theta Y_{i j}+\sin \theta Y_{i^{\prime} j}$. There follows:

$$
\begin{aligned}
\cos & \theta \partial_{i}\left[g_{j} \circ \rho\right]+\sin \theta \partial_{i^{\prime}}\left[g_{j} \circ \rho\right]=\left[\partial_{i} g_{j}\right] \circ \rho=\left[Y_{i j} \tilde{f}\right] \circ \rho \\
& =\cos \theta Y_{i j}[\tilde{f} \circ \rho]+\sin \theta Y_{i^{\prime} j}[\tilde{f} \circ \rho] \\
& =\cos \theta Y_{i j} \tilde{f}+\sin \theta Y_{i^{\prime} j} \tilde{f}=\cos \theta \partial_{i} g_{j}+\sin \theta \partial_{i^{\prime}} g_{j},
\end{aligned}
$$

and this implies $g_{j} \circ \rho=g_{j}$, since the operator $\cos \theta \partial_{i}+\sin \theta \partial_{i^{\prime}}$ is one-to-one in $B_{0}$.

We now show that there exists a $G_{1}$-invariant hyperfunction $\varphi \in B_{0}$ such that $Y_{i j} \varphi=Y_{i j} \widetilde{f}$ for some fixed $i$ and $j, 1 \leqslant i \leqslant p$ and $p+1 \leqslant j \leqslant p+q$. We have to distinguish two cases:

(A) If $q$ is equal to one. $G_{1}$ is then the group generated by the $X_{i i}{ }^{\prime}$ $\left(1 \leqslant i<i^{\prime} \leqslant p\right)$. It follows from Remark 9, applied to the $p$ first variables only, that every $G_{1}$-invariant hyperfunction supported at the origin can be written

$$
\varphi=\sum_{\alpha, \beta \in \mathbb{N}} b_{\alpha \beta} \Theta^{\alpha} \partial_{j}^{\beta} \delta \text { where } \Theta=\partial_{1}^{2}+\cdots+\partial_{p}^{2}=\partial_{i}^{2}+\Theta_{0} .
$$

We have thus:

$$
\begin{aligned}
Y_{i j} \varphi & =\left(x_{i} \partial_{j}+x_{j} \partial_{i}\right)\left(\sum_{\alpha, \beta} b_{\alpha \beta} \Theta^{\alpha} \partial_{j}^{\beta} \delta\right) \\
& =\sum_{\alpha, \beta} b_{\alpha \beta}\left\{-\left[\sum_{0<k<\alpha} 2 k\left(\begin{array}{l}
\alpha \\
k
\end{array}\right) \partial_{i}^{2 k-1} \Theta_{0}^{\alpha-k}\right] \partial_{j}^{\beta+1}-\beta \Theta^{\alpha} \partial_{i} \partial_{j}^{\beta-1}\right\} \delta \\
& =-\partial_{i} \sum_{\alpha, \beta} b_{\alpha \beta}\left(2 \alpha \Theta^{\alpha-1} \partial_{j}^{\beta+1}+\beta \Theta^{\alpha} \partial_{j}^{\beta-1}\right) \delta .
\end{aligned}
$$

Since $g_{j}$ is $G_{1}$-invariant, it can also be written

$$
g_{j}=\sum_{\alpha, \beta} c_{\alpha \beta} \Theta^{\alpha} \partial_{j}^{\beta} \delta
$$


with the convergence condition $\left(C^{\prime \prime}\right)$ on the coefficients $c_{\alpha \beta}$ (see Proposition 8 and Remark 9):

$$
\limsup _{2 \alpha+\beta \rightarrow \infty}\left[\left|c_{\alpha \beta}\right|(2 \alpha) ! \beta !\right]^{1 /(2 \alpha+\beta)}=0 .
$$

Thus we are led to solve the equation (with unknown $b_{\alpha \beta}$ )

$$
\sum b_{\alpha \beta}\left(2 \alpha X^{\alpha-1} Y^{\beta+1}+\beta X^{\alpha} Y^{\beta-1}\right)=\sum c_{\alpha \beta} X^{\alpha} Y^{\beta}
$$

in the space of series of two indeterminates satisfying $\left(C^{\prime \prime}\right)$, that is

$$
\begin{aligned}
& \sum b_{\alpha, 2 \beta}\left(2 \alpha X^{\alpha-1}\left(Y^{2}\right)^{\beta}+\beta X^{\alpha}\left(Y^{2}\right)^{\beta-1}\right) Y=\sum c_{\alpha, 2 \beta+1} X^{\alpha}\left(Y^{2}\right)^{\beta} Y . \\
& \sum b_{\alpha, 2 \beta-1}\left(2 \alpha X^{\alpha-1}\left(Y^{2}\right)^{\beta}+\beta X^{\alpha}\left(Y^{2}\right)^{\beta-1}\right)=\sum c_{\alpha, 2 \beta} X^{\alpha}\left(Y^{2}\right)^{\beta} .
\end{aligned}
$$

Condition $\left(C^{\prime \prime}\right)$ is easily seen to be invariant under translation on one of the indices (in fact $\left(C^{\prime \prime}\right)$, which means that $\Sigma c_{\alpha \beta} \delta^{(2 \alpha, 2 \beta)}$ converges to a hyperfunction of two variables supported at the origin, is easily seen to be equivalent to the statement that the function of two complex variables $\Sigma c_{\alpha \beta} x^{2 \alpha} y^{2 \beta}$ is an entire function of zero exponential type), so that we are led to solve twice an equation of the type

$$
\sum f_{\alpha \beta}\left(2 \alpha X^{\alpha-1} Z^{\beta}+\beta X^{\alpha} Z^{\beta-1}\right)=\sum g_{\alpha \beta} X^{\alpha} Z^{\beta}
$$

in the space of series of two indeterminates $X$ and $Z$ satisfying $\left(C^{\prime \prime}\right)$. The last equation can be written

$$
\left(2 \partial_{X}+\partial_{Z}\right)\left(\sum f_{\alpha \beta} X^{\alpha} Z^{\beta}\right)=\sum g_{\alpha \beta} X^{\alpha} Z^{\beta},
$$

and transforms by the change of indeterminates $X=2 U, Z=U+V$, into

$$
\partial_{U}\left(\sum f_{\alpha \beta}^{\prime} U^{\alpha} V^{\beta}\right)=\sum g_{\alpha \beta}^{\prime} U^{\alpha} V^{\beta}
$$

where the new coefficients $f_{\alpha \beta}^{\prime}, g_{\alpha \beta}^{\prime}$ must satisfy some condition, the transform of $\left(C^{\prime \prime}\right)$. If we prove that this new condition is no other than $\left(C^{\prime \prime}\right)$, then it is obvious that any such equation can be solved in the required space (choosing for instance $f_{0 \beta}^{\prime}=0$ and $f_{\alpha \beta}^{\prime}=\alpha^{-1} g_{\alpha-1, \beta}^{\prime}$ for $\alpha>0$ ), and the proof will be complete in case $\mathbf{A}$.

Let us prove first that if the new coefficients satisfy $\left(C^{\prime \prime}\right)$, then the old ones do as well. We put

$$
F=\sum f_{\alpha \beta}^{\prime} U^{\alpha} V^{\beta}=\sum f_{\alpha \beta}^{\prime}(X / 2)^{\alpha}(Z-X / 2)^{\beta} .
$$


As condition ( $\left.C^{\prime \prime}\right)$ is obviously invariant under the change $X / 2 \mapsto X$, we take the liberty to omit the two's in the following, so that we write

$$
\begin{aligned}
F & =\sum_{\alpha, \beta} f_{\alpha \beta}^{\prime}\left(\sum_{j=0}^{\beta}(-1)^{\beta-j}\left(\begin{array}{l}
\beta \\
j
\end{array}\right) X^{\alpha+\beta-j} Z^{j}\right) \\
& =\sum_{\gamma, j}\left(\sum_{k=0}^{\gamma}(-1)^{k}\left(\begin{array}{c}
j+k \\
j
\end{array}\right) f_{\gamma-k, j+k}^{\prime}\right) X^{\gamma} Z^{j}=\sum_{\gamma, j} f_{\gamma, j} X^{\gamma} Z^{j} .
\end{aligned}
$$

Condition $\left(\mathrm{C}^{\prime \prime}\right)$ is equivalent to saying that for every $\epsilon>0$ there exists a constant $C>0$ such that, for any integers $r, s,\left|f_{r s}^{\prime}\right| \leqslant C \epsilon^{r+s} /(2 r) !(2 s)$ !, but then

$$
\begin{aligned}
\left|f_{\gamma, j}\right| & \leqslant C \sum_{k=0}^{\gamma}\left(\begin{array}{c}
j+k \\
j
\end{array}\right) \frac{\epsilon^{\gamma+j}}{(2 \gamma-2 k) !(2 j+2 k) !} \\
& \leqslant C \sum_{k=0}^{\gamma}\left(\begin{array}{c}
2 j+2 k \\
2 j
\end{array}\right) \frac{\epsilon^{\gamma+j}}{(2 \gamma-2 k) !(2 j+2 k) !} \\
& \leqslant C\left(\sum_{k=0}^{\gamma} \frac{(2 \gamma) !}{(2 k) !(2 \gamma-2 k) !}\right) \frac{\epsilon^{\gamma+j}}{(2 \gamma) !(2 j) !} \leqslant \frac{C(4 \epsilon)^{\gamma+j}}{(2 \gamma) !(2 j) !}
\end{aligned}
$$

and this means that the $f_{\gamma, j}$ also satisfy $\left(C^{\prime \prime}\right)$. It is obvious from the proof that it works for any linear substitution of indeterminates, so that we will not write it for the other implication.

(B) If $q$ is larger than one. We note first that the analogue of the first part of the proof, the roles of the indices $i$ and $j$ being exchanged, would show that there also exists a hyperfunction $h_{i} \in B_{0}$ such that $Y_{i j} \tilde{f}=\partial_{j} h_{i}$ for every $j, p+1 \leqslant j \leqslant p+q$, and that $h_{i}$ is independent of $j$ and invariant under the rotations on the variables $x_{j}(p+1 \leqslant j \leqslant p+q)$, so that finally $Y_{i j} \tilde{f}=\partial_{i} \partial_{j} k$ where $k \in B_{0}$ is independent of both $i$ and $j$, and invariant under the rotations on the variables $x_{i}(1 \leqslant i \leqslant p)$ as well as those on the variables $x_{j}(p+1 \leqslant j \leqslant p+q)$, that is to say under the whole group $G_{1}$.

Remark 9, applied separately to the $p$ first and to the $q$ last variables, shows that any $G_{1}$-invariant hyperfunction $\varphi \in B_{0}$ can be written

$$
\varphi=\sum_{\alpha, \beta \in N} b_{\alpha \beta} \Theta^{\alpha} \Xi^{\beta} \delta
$$

where $\Theta=\partial_{1}^{2}+\cdots+\partial_{p}^{2}=\partial_{i}^{2}+\Theta_{0}$ and $\Xi=\partial_{p+1}^{2}+\cdots+\partial_{p+q}^{2}=$ $\partial_{j}^{2}+\Xi_{0}$. In particular $k=\Sigma_{\alpha, \beta \in N} c_{\alpha \beta} \Theta^{\alpha} \Xi^{\beta} \delta$. But 


$$
\begin{aligned}
& Y_{i j} \varphi=\left(x_{i} \partial_{j}+x_{j} \partial_{i}\right)\left(\sum_{\alpha, \beta} b_{\alpha \beta} \Theta^{\alpha} \Xi^{\beta} \delta\right) \\
&= \sum_{\alpha, \beta} b_{\alpha \beta}\left\{\left[\sum_{0<k<\alpha}-2 k\left(\begin{array}{l}
\alpha \\
k
\end{array}\right) \partial_{i}^{2 k-1} \Theta_{0}^{\alpha-k}\right] \Xi^{\beta} \partial_{j}\right. \\
&\left.+\partial_{i} \Theta^{\alpha}\left[\sum_{0<l<\beta}-2 l\left(\begin{array}{l}
\beta \\
l
\end{array}\right) \partial_{j}^{2 l-1} \Xi_{0}^{\beta-l}\right]\right\} \delta \\
&=-2 \partial_{i} \partial_{j} \sum_{\alpha, \beta} b_{\alpha \beta}\left(\alpha \Theta^{\alpha-1} \Xi^{\beta}+\beta \Theta^{\alpha} \Xi^{\beta-1}\right) \delta .
\end{aligned}
$$

We are led now to solve the equation (in the unknown $b_{\alpha \beta}$ )

$$
\sum b_{\alpha \beta}\left(\alpha X^{\alpha-1} Y^{\beta}+\beta X^{\alpha} Y^{\beta-1}\right)=\sum c_{\alpha \beta} X^{\alpha} Y^{\beta}
$$

in the space of two indeterminates whose coefficients satisfy the same condition $\left(C^{\prime \prime}\right)$ as in the preceding case. This equation can be written

$$
\left(\partial_{X}+\partial_{Y}\right)\left(\sum b_{\alpha \beta} X^{\alpha} Y^{\beta}\right)=\sum c_{\alpha \beta} X^{\alpha} Y^{\beta}
$$

and is easily seen to have a solution by the change of indeterminates $X+Y=U$, $X-Y=V$, which leaves $\left(\mathrm{C}^{\prime \prime}\right)$ invariant as in case $\mathrm{A}$, and transforms the equation into

$$
\partial_{U}\left(\sum b_{\alpha \beta}^{\prime} U^{\alpha} V^{\beta}\right)=\sum c_{\alpha \beta}^{\prime} U^{\alpha} V^{\beta}
$$

$b_{0 \beta}^{\prime}=0$ and $b_{\alpha \beta}^{\prime}=\alpha^{-1} c_{\alpha-1, \beta}^{\prime}$ for $\alpha>0$, is an obvious solution.

We conclude that there exists a $G_{1}$-invariant hyperfunction $\varphi \in B_{0}$ such that $Y_{i j} \varphi=Y_{i j} \widetilde{f}$ for some fixed integers $i$ and $j, 1 \leqslant i \leqslant p$ and $p+1 \leqslant j$ $\leqslant p+q$.

Since both $\varphi$ and $\tilde{f}$ are $G_{1}$-invariant, we have

$$
\begin{gathered}
\forall i^{\prime}, i^{\prime \prime}, 1 \leqslant i^{\prime} \neq i^{\prime \prime} \leqslant p, \quad \forall j^{\prime}, j^{\prime \prime}, p+1 \leqslant j^{\prime} \neq j^{\prime \prime} \leqslant p+q, \\
X_{i^{\prime} i^{\prime \prime} \varphi}=X_{i^{\prime} i^{\prime \prime}} \tilde{f}=X_{j^{\prime} j^{\prime \prime} \varphi}=X_{j^{\prime} j^{\prime \prime}} \widetilde{f}=0 .
\end{gathered}
$$

Using this and the commutation relations (2), we get

$$
\begin{aligned}
Y_{i^{\prime} j^{\prime}} \varphi & =\left[X_{i^{\prime} t}, Y_{i j^{\prime}}\right] \varphi=X_{i^{\prime} i} Y_{i j^{\prime} \varphi} \\
& =X_{i^{\prime} i}\left[X_{j^{\prime} j}, Y_{i j}\right] \varphi=X_{i^{\prime} t^{\prime}} X_{j^{\prime} j} Y_{i j} \varphi \\
& =X_{i^{\prime} i} X_{j^{\prime} j} Y_{i j} \widetilde{f}=X_{i^{\prime} i} Y_{i j^{\prime}} \widetilde{f}=Y_{i^{\prime} j^{\prime}} \widetilde{f}
\end{aligned}
$$


for all $i^{\prime}, i^{\prime \prime}, j^{\prime}, j^{\prime \prime}$ such that $1 \leqslant i \neq i^{\prime} \leqslant p$ and $p+1 \leqslant j \neq j^{\prime} \leqslant p+q$. But this means that $\bar{f}=\tilde{f}-\varphi$ is such that

$$
X_{i^{\prime} i^{\prime \prime}} \bar{f}=X_{j^{\prime} j^{\prime \prime}} \bar{f}=Y_{i^{\prime} j^{\prime}} \bar{f}=0 ;
$$

that is to say $\bar{f}$ is $G_{0}$-invariant on $\mathbf{R}^{p+q}$. Finally

$$
\left.\bar{f}\right|_{R^{p+q_{-}}\{0\}}=\left.\widetilde{f}\right|_{R^{p+q_{-}}-\{0\}}=f \text { Q.E.D. }
$$

COROLlaRY 11. Every G-invariant hyperfunction on $\mathbf{R}^{p+q}-\{0\}$ can be extended to a G-invariant hyperfunction on $\mathrm{R}^{p+q}$.

Proof. Theorem 10 gives a $G_{0}$-invariant extension. As $G$ is the semidirect product of $G_{0}$ by a finite group $H$, it is enough to select the symmetric part of this extension with respect to $H$.

REMARK. If $p q>1$, any $G$ - or $G_{0}$-invariant hyperfunction on any stable open set of $\mathbf{R}^{p+q}$ can be extended to an invariant hyperfunction on any larger stable open set: it is a consequence of Theorem 10 and Corollary 11 when one wants an extension at the origin, and of the flabbiness of sheaf $B$ (and Propositions 5 and 7) when one wants to extend elsewhere.

2. Hyperfunctions on $\mathbf{R}^{\mathbf{2}}$ invariant under a noncompact orthogonal group. In this section, we study the hyperfunctions on $\mathbf{R}^{\mathbf{2}}$ invariant under the orthogonal group of a nondegenerate nondefinite quadratic form $Q$, or under its connected component of the identity, say $G$ and $G_{0}$. It will be handy to choose coordinates $(x, y)$ in which the quadratic form is written

$$
Q(x, y)=x y \text {. }
$$

$G$ is then also the subgroup of $G L(2, R)$ which leaves invariant the operator $\Pi=\partial_{x y}^{2}$, and $G_{0}$ is the group of matrices

$$
\left(\begin{array}{cc}
a & 0 \\
0 & 1 / a
\end{array}\right) \quad(a>0)
$$

Contrary to the preceding case (Theorem 10 ), there are $G_{0}$-invariant hyperfunctions on $\mathbf{R}^{2}-\{0\}$ which refuse to extend to $G_{0}$-invariant hyperfunctions on $\mathbf{R}^{2}$. Let us give immediately an example:

We write $1 /|x|$ for the distribution on $R$ defined by

$$
\varphi \in C_{c}^{\infty}(\mathbf{R}) \mapsto \lim _{\epsilon \rightarrow 0}\left[-\int_{-\infty}^{-\epsilon} \frac{\varphi(x)}{x} d x+\int_{\epsilon}^{+\infty} \frac{\varphi(x)}{x} d x+2 \varphi(0) \ln \epsilon\right],
$$

which satisfies $x \cdot 1 / 2|x|=1 / 2 \operatorname{sgn}(x)=H(x)-1 / 2$, where $H$ is the Heaviside function. 
Proposition 12. Let $T$ be the restriction to $\mathbf{R}^{2}-\{0\}$ of the distribution $1 / 2|x| \otimes \delta(y) . T$ is $G_{0}$-invariant. There is no $G_{0}$-invariant hyperfunction on $\mathbf{R}^{2}$ whose restriction to $\mathbf{R}^{2}-\{0\}$ is $T$.

PRoof. $G_{0}$ is connected, and its infinitesimal generator (on $R^{2}$ ) is a multiple of $x \partial_{x}-y \partial_{y}$. A hyperfunction $f$ (on $\mathbf{R}^{2}$ or $\mathbf{R}^{2}-\{0\}$ ) is thus $G_{0}$-invariant, according to Lemma 1 , if and only if $\left(x \partial_{x}-y \partial_{y}\right) f=0$. But

$$
\begin{aligned}
\left(x \partial_{x}-y \partial_{y}\right)(1 / 2|x| \otimes \delta(y)) & =\left(\partial_{x}(x \cdot)-\partial_{y}(y \cdot)\right)(1 / 2|x| \otimes \delta(y)) \\
& =\partial_{x}((H(x)-1 / 2) \otimes \delta(y))=\delta(x, y) .
\end{aligned}
$$

And by restriction to $\mathbf{R}^{2}-\{0\}:\left(x \partial_{x}-y \partial_{y}\right) T=0$. Assume there is $\widetilde{T} \in B\left(\mathbf{R}^{2}\right)$, $G_{0}$-invariant and such that $\left.\widetilde{T}\right|_{R^{2}-\{0\}}=T$, and put $S=1 / 2|x| \otimes \delta(y)-\widetilde{T} . S$ is supported at the origin, thus it can be written

$$
S=\sum_{\alpha, \beta \in \mathbf{N}} a_{\alpha \beta} \delta^{(\alpha, \beta)}
$$

with

$$
\limsup _{\alpha+\beta \rightarrow \infty}\left(\left|a_{\alpha \beta}\right| \alpha ! \beta !\right)^{1 /(\alpha+\beta)}=0 .
$$

Thus $\left(x \partial_{x}-y \partial_{y}\right) S=\Sigma_{\alpha, \beta} a_{\alpha \beta}(\beta-\alpha) \delta^{(\alpha, \beta)}$ but this contradicts

$$
\left(x \partial_{x}-y \partial_{y}\right) S=\left(x \partial_{x}-y \partial_{y}\right)(1 / 2|x| \otimes \delta(y))=\delta . \quad \text { Q.E.D. }
$$

In fact the proof leads us to a first result:

- Proposition 13. Let $f$ be a $G_{0}$-invariant hyperfunction on $\mathbf{R}^{2}-\{0\}$, and $\tilde{f}$ an arbitrary extension of $f$ on $\mathbf{R}^{2}$. The hyperfunction $\left(x \partial_{x}-y \partial_{y}\right) \tilde{f}$ is a series of derivatives of the Dirac measure $\delta$. $f$ has a $G_{0}$-invariant extension on $\mathbf{R}^{2}$ if and only if all the diagonal coefficients in this series vanish.

Proof. The necessity of the condition is implied by the preceding proof. If, on the other hand, $\left(x \partial_{x}-y \partial_{y}\right) \tilde{f}=\Sigma_{\alpha, \beta} a_{\alpha \beta} \delta^{(\alpha, \beta)}$, with $a_{\alpha \alpha}=0$, choose $g=\Sigma_{\alpha \neq \beta} b_{\alpha \beta} \delta^{(\alpha, \beta)}$, with $b_{\alpha \beta}=a_{\alpha \beta} /(\beta-\alpha)$. Since $\left|b_{\alpha \beta}\right| \leqslant\left|a_{\alpha \beta}\right|$, the last series is convergent in $B_{0}$. Putting $\bar{f}=\tilde{f}-g$, one gets $\left(x \partial_{x}-y \partial_{y}\right) \bar{f}=0$ and $\left.\bar{f}\right|_{R^{2}-\{0\}}=\left.\widetilde{f}\right|_{R^{2}-\{0\}}=f$. Q.E.D.

REMARK. T. Miwa proved [5, Proposition 3.1] that $\left(x \partial_{x}-y \partial_{y}\right): B\left(\mathbf{R}^{2}\right)$ $\rightarrow B\left(\mathbf{R}^{2}\right)$ is onto. Thus Lemma 2 applies here with $M=\mathbf{R}^{2}, U=\mathbf{R}^{2}-\{0\}$, $F=\{0\}, X=x \partial_{x}-y \partial_{y}$, for the group $G_{0}$. But the last proposition exhibits a complement of $X B_{\{0\}}\left(R^{2}\right)$ in $B_{\{0\}}\left(R^{2}\right)$ : the hyperfunctions which have the form

$$
\sum a_{\alpha} \delta^{(\alpha, \alpha)}=\sum a_{\alpha}\left(\partial_{x y}^{2}\right)^{\alpha} \delta
$$


(with the convergence condition $\lim \sup _{\alpha \rightarrow \infty}\left(\left|a_{\alpha}\right|(2 \alpha) !\right)^{1 / 2 \alpha}=0$; see Proposition 8). Thus Lemma 2 and Proposition 13 allow us to construct an explicit complement of $\pi B^{G_{0}}\left(\mathbf{R}^{2}\right)$ in $B^{G_{0}}\left(\mathbf{R}^{2}-\{0\}\right)$ : it is enough for that to note that $\partial_{x y}^{2}$ and $X$ commute, since the first is $G_{0}$-invariant. But we can proceed directly:

Now put $T=1 / 4|x| \otimes \delta(y)-\delta(x) \otimes 1 / 4|y|$; as $X$ is antisymmetric in $x$ and $y$, one still has $X T=\delta$, and $X\left(\partial_{x y}^{2}\right)^{\alpha} T=\left(\partial_{x y}^{2}\right)^{\alpha} X T=\delta^{(\alpha, \alpha)} \quad(\alpha \in \mathrm{N})$.

LEMMA 14. If $\Sigma a_{\alpha} \delta^{(\alpha, \alpha)}$ is a hyperfunction supported at the origin, the series $S_{a}=\Sigma a_{\alpha}\left(\partial_{x y}^{2}\right)^{\alpha} T$ defines a hyperfunction on $\mathbf{R}^{2}$ such that $X S_{a}=$ $\Sigma a_{\alpha} \delta^{(\alpha, \alpha)}$.

Proof. It is clear if one knows that the convolution product of two hyperfunctions enjoys the same properties as that of distributions. We give here a direct proof.

As the variables $x$ and $y$ play antisymmetric roles, it is obviously enough to prove that $\bar{S}_{a}=\Sigma a_{\alpha}\left(\partial_{x y}^{2}\right)^{\alpha} \bar{T}$ has the same properties, where $\bar{T}=$ $1 / 2|x| \otimes \delta(y)$. Noting ln, the principal determination of the logarithm, holomorphic except on the negative real half-line, one has

$$
1 / 2|x|=-(1 / 4 i \pi)[(1 / \xi)(\ln \xi+\ln (-\xi))]_{\xi=x}
$$

and

$$
\bar{T}=-\left(1 / 8 \pi^{2}\right)[(1 / \xi \eta)(\ln \xi+\ln (-\xi))]
$$

where the bracket is a relative 2-cocycle, represented by a function holomorphic at least in $(\mathbf{C}-\mathbf{R})^{2}$. If the series

$$
\sum a_{\alpha}\left(\partial_{\xi \eta}^{2}\right)^{\alpha}\{(1 / \xi \eta)(\ln \xi+\ln (-\xi))\}
$$

is uniformly convergent on every compact subset of $(\mathbf{C}-\mathbf{R})^{2}$, it will define a function holomorphic in $(\mathbf{C}-\mathbf{R})^{2}$, termwise differentiable, thus a relative 2-cocycle representing a hyperfunction $-8 \pi^{2} \bar{S}_{a}$ on $\mathbf{R}^{2}$, and $\bar{S}_{a}$ will obviously have the desired property. But

$$
\left(\partial_{\xi \eta}^{2}\right)^{\alpha}\left\{\frac{1}{\xi \eta}(\ln \xi+\ln (-\xi))\right\}=\frac{(\alpha !)^{2}}{(\xi \eta)^{\alpha+1}}\left\{-\frac{2}{\alpha}\left(\sum_{j=1}^{\alpha} \frac{1}{j}\right)+\ln \xi+\ln (-\xi)\right\} .
$$

Since $\Sigma_{j=1}^{\alpha} 1 / j \sim \ln \alpha \ll \alpha$, it is clearly enough to prove that the series $\Sigma a_{\alpha}(\alpha !)^{2} /(\xi \eta)^{\alpha+1}$ is uniformly convergent on every compact subset of $(C-\{0\})^{2}$; but this is precisely saying that

$$
\sum a_{\alpha} \delta^{(\alpha, \alpha)}=-\frac{1}{4 \pi^{2}}\left[\sum a_{\alpha} \frac{(\alpha !)^{2}}{(\xi \eta)^{\alpha+1}}\right]
$$


defines a hyperfunction supported at the origin. Q.E.D.

We now state the analogues of Propositions 5, 7 and 8 of $\S 1$.

Proposition 15. The $G_{0}$-invariant hyperfunctions supported at the origin have the form $\Sigma_{\alpha \in N} a_{\alpha}\left(\partial_{x y}^{2}\right)^{\alpha} \delta$, with

$$
\limsup _{\alpha \rightarrow \infty}\left(\left|a_{\alpha}\right|(2 \alpha) !\right)^{1 / 2 \alpha}=0 .
$$

All these are G-invariant.

PROoF. If $f=\Sigma_{\alpha, \beta \in N} a_{\alpha \beta} \partial_{x}^{\alpha} \partial_{y}^{\beta} \delta$ is $G_{0}$-invariant, one has

$$
0=\left(x \partial_{x}-y \partial_{y}\right) f=\sum a_{\alpha \beta}(\beta-\alpha) \partial_{x}^{\alpha} \partial_{y}^{\beta} \delta
$$

and $a_{\alpha \beta}$ vanishes as soon as $\alpha \neq \beta$. Thus $f$ has the required form, and ( $\left.C^{\prime}\right)$ can be proved as in Proposition 8. Again, the last statement is obvious, since $\partial_{x y}^{2}$ and $\delta$ are $G$-invariant. Q.E.D.

Let $Q$ be the analytic map from $\mathrm{R}^{2}-\{0\}$ into $\mathrm{R}: Q(x, y)=x y$.

Proposition 16. A hyperfunction on $\mathbf{R}^{2}-\{0\}$ is G-invariant if and only if it is the inverse image under $Q$ of some hyperfunction on $\mathbf{R}$.

Apart from the change of coordinates $x=x_{1}+x_{2}, y=x_{1}-x_{2}$, the proof is analogous to that of Proposition 5, but it is useful to recall the beginning of it here: in any of the half-spaces $E_{1}=\{x>0\}$ or $E_{3}=\{x<0\}$ one can choose $x y$ and $x$ as new coordinates, while one can choose $x y$ and $y$ in $E_{2}=\{y>0\}$ or $E_{4}=\{y<0\}$. If $f$ is $G_{0}$-invariant, $\left.f\right|_{E_{j}}$ depends only on $x y$, and thus it is the inverse image of a hyperfunction $T_{j}$ on $\mathbf{R}(j=1, \cdots, 4)$. As this inverse image is obviously injective, one has necessarily:

$$
\begin{array}{ll}
\left.T_{1}(v)\right|_{v>0}=\left.T_{2}(v)\right|_{v>0}, & \left.T_{1}(v)\right|_{v<0}=\left.T_{4}(v)\right|_{v<0,}, \\
\left.T_{3}(v)\right|_{v<0}=\left.T_{2}(v)\right|_{v<0}, & \left.T_{3}(v)\right|_{v>0}=\left.T_{4}(v)\right|_{v>0 .}
\end{array}
$$

Let now $\mathbf{R}^{\prime \prime}$ be the non-Hausdorff manifold obtained by taking four copies of $\mathbf{R}$, say $\mathbf{R}_{1}, \mathbf{R}_{2}, \mathbf{R}_{3}, \mathbf{R}_{4}$, and sticking together the positive parts of $\mathbf{R}_{1}$ and $\mathbf{R}_{\mathbf{2}}$ and of $\mathbf{R}_{3}$ and $\mathbf{R}_{4}$, and the negative parts of $\mathbf{R}_{1}$ and $\mathbf{R}_{4}$ and of $\mathbf{R}_{2}$ and $\mathbf{R}_{3}$. A hyperfunction $T$ on $\mathbf{R}^{\prime \prime}$ will be a family of four hyperfunctions $T_{i}$ $(j=1, \cdots, 4)$ on $\mathbf{R}$ satisfying the above conditions. Finally let us define an analytic map $Q^{\prime \prime}$ from $\mathbf{R}^{2}-\{0\}$ into $\mathbf{R}^{\prime \prime}$, by the condition that it coincides on each $E_{j}$ with $Q: E_{j} \rightarrow \mathbf{R}_{j}$. The inverse image under $Q^{\prime \prime}$ of a hyperfunction on $\mathbf{R}^{\prime \prime}$ is the hyperfunction on $\mathbf{R}^{2}-\{0\}$ defined in the obvious way.

PRoposition 17. The $G_{0}$-invariant hyperfunctions on $\mathbf{R}^{2}-\{0\}$ are the inverse images under $Q^{\prime \prime}$ of the hyperfunctions on $\mathbf{R}^{\prime \prime}$. 
Proof. We just proved the necessity. The sufficiency is obvious.

To state the next result, we need a few notations. First $G / G_{0}$ has four elements, and two generators are represented by

the symmetry $\sigma$ exchanging $x$ and $y$,

the symmetry $\tau$ exchanging $x$ and $-y$.

If $f \in B\left(R^{2}-\{0\}\right)$ is $G_{0}$-invariant, one can decompose it in a unique way into a sum $f=f_{0}+f_{\sigma}+f_{\tau}+f_{\sigma \tau}$ where each term is $G_{0}$-invariant and such that

$$
\begin{array}{ll}
f_{0} \circ \sigma=f_{0} \circ \tau=f_{0}, & f_{\sigma} \circ \sigma=-f_{\sigma}, f_{\sigma} \circ \tau=f_{\sigma}, \\
f_{\tau} \circ \sigma=f_{\tau}, & f_{\tau} \circ \tau=-f_{\tau}, f_{\sigma \tau} \circ \sigma=f_{\sigma \tau} \circ \tau=-f_{\sigma \tau} .
\end{array}
$$

We call $f_{\sigma \tau}$ the antisymmetric part of $f$.

If now $T=\left(T_{1}, T_{2}, T_{3}, T_{4}\right)$ is a hyperfunction on $\mathrm{R}^{\prime \prime}$, we call mass of $T$ the hyperfunction (supported at the origin) $T_{1}-T_{2}+T_{3}-T_{4}$.

THEOREM 18. Let $f \in B\left(\mathbf{R}^{2}-\{0\}\right)$ be $G_{0}$-invariant, and $T \in B\left(\mathbf{R}^{\prime \prime}\right)$ such that $f=Q^{n-1}(T)$. The following conditions are equivalent:

(1) $f$ has $a G_{0}$-invariant extension at the origin.

(2) The antisymmetric part of $f$ is zero.

(3) The mass of $T$ is zero.

REMARK. We will see further two other conditions equivalent to these: see Remark 21 and Corollary 23.

Proof. (2) $\Leftrightarrow$ (3). As $f_{\sigma \tau}=1 / 4(f-f \circ \sigma-f \circ \tau+f \circ \sigma \circ \tau)$, it is easily seen that, in any open half-space $E_{j}, f_{\sigma \tau}$ is the inverse image of $1 / 4\left(T_{1}-T_{2}+T_{3}-T_{4}\right)$.

(2) $\Rightarrow(1)$. If $f_{\sigma \tau}=0, f$ is the sum of three hyperfunctions invariant by at least one of the symmetries $\sigma$ or $\tau$. Let $g$ be one of these, symmetric in $\boldsymbol{x}$ and $\pm y$, and $\widetilde{g}$ an extension of $\boldsymbol{g}$ at the origin. We can assume that $\widetilde{\boldsymbol{g}}$ has the same symmetry property. But then $\left(x \partial_{x}-y \partial_{y}\right) \widetilde{g}$ is changed into its opposite by the exchange of $x$ and $\pm y$, and this excludes the possibility that its development in a series of derivatives of $\delta$ might contain nontrivial diagonal terms. We conclude by Proposition 13.

non (2) $\Rightarrow$ non (1). One can assume $f=f_{\sigma \tau}$. In the first quadrant $E_{1} \cap E_{2}$ for example, $\sigma$ transforms $Q^{-1}\left(T_{1}\right)$ into $Q^{-1}\left(T_{2}\right)=Q^{-1}\left(T_{1}\right)$, so that $\sigma$ keeps $f$ unchanged. This implies $\left.f\right|_{E_{1} \cap E_{2}}=0$. One could reason in the same way in $E_{3} \cap E_{4}$, and also in the two other quadrants by considering the symmetry $\tau$. Thus $f$ is supported on the two axes. In the neighborhood of a point on the half-axis $\{y=0, x>0\}$ for instance, $f$ can thus be written

$$
f(x, y)=\sum_{\alpha \in N} f_{\alpha}(x) \otimes \delta^{(\alpha)}(y) .
$$


Since $\delta^{(\alpha)}(y)$ is homogeneous on $\mathbf{R}$, of degree $-\alpha-1$, and $f$ is invariant under the group $G_{0}$ of matrices $\left(\begin{array}{cc}a & 0 \\ 0 & 1 / a\end{array}\right) \quad(a>0), f_{\alpha}(x)$ must be positively homogeneous of degree $-\alpha-1$ :

$$
f_{\alpha}(x)=(-1)^{\alpha} \alpha ! a_{\alpha} / 4 x^{\alpha+1}
$$

for every $x>0$, and for some constant $a_{\alpha}$. As $f$ is changed into its opposite by $\sigma$ and by $\tau$, there follows

$$
\begin{aligned}
f(x, y)= & \sum_{\alpha \in N} \frac{(-1)^{\alpha} \alpha ! a_{\alpha} \operatorname{sgn}(x)}{4 x^{\alpha+1}} \otimes \delta^{(\alpha)}(y) \\
& -\sum_{\alpha \in \mathbb{N}} \delta^{(\alpha)}(x) \otimes \frac{(-1)^{\alpha} \alpha ! a_{\alpha} \operatorname{sgn}(y)}{4 y^{\alpha+1}}
\end{aligned}
$$

on $\mathbf{R}^{2}-\{0\}$, and that is to say:

$$
f(x, y)=\left.\sum_{\alpha \in N} a_{\alpha}\left(\partial_{x y}^{2}\right)^{\alpha} T\right|_{\mathrm{R}^{2}-\{0\}}=\left.S_{a}\right|_{\mathrm{R}^{2}-\{0\}}
$$

with the notations of Lemma 14.

We can apply Proposition 13 to the extension $S_{a}$ of $f$ : since, according to Lemma 14, $\left(x \partial_{x}-y \partial_{y}\right) S_{a}=\Sigma_{\alpha \in N} a_{\alpha} \delta^{(\alpha, \alpha)}, f$ has a $G_{0}$-invariant extension at the origin if and only if $a_{\alpha}=0$ for every $\alpha$, that is, if $f=0$. Q.E.D.

COROLlaRY 19. Every G-invariant hyperfunction on $\mathbf{R}^{2}-\{0\}$ can be extended to a G-invariant hyperfunction on $\mathbf{R}^{2}$.

Proof. To say that $f$ is $G$-invariant is to say that $f=f_{0}$. Thus $f_{\sigma \tau}=0$, and Theorem 18 gives a $G_{0}$-invariant extension $\tilde{f}$. A $G$-invariant extension is then $1 / 4(\tilde{f}+\tilde{f} \circ \sigma+\tilde{f} \circ \tau+\tilde{f} \circ \sigma \circ \tau)$. Q.E.D.

We now take a closer look at the hyperfunctions on $\mathbf{R}^{\prime \prime}$, and particularly at the ones "without mass" (that is such that their mass is zero).

If $T=\left(T_{1}, T_{2}, T_{3}, T_{4}\right) \in B\left(R^{\prime \prime}\right)$, we define the shuttle integral of $T$ (and we write $\oint T$ ) in the following way: let $K_{j}$ be a compact set in $\mathbf{R}_{j}$, containing the origin as an interior point, and $F_{j}$ the closure of the complement of $K_{j}$ in $\mathbf{R}_{j}$. The hyperfunction $T_{j}$ can be written as the sum of two hyperfunctions, $T_{j}^{1}$ supported in $K_{j}$ and $T_{j}^{2}$ supported in $F_{j}$; we put:

$$
\oint T(v) d v=\int_{\mathrm{R}_{1}} T_{1}^{1}(v) d v-\int_{\mathrm{R}_{2}} T_{2}^{1}(v) d v+\int_{\mathrm{R}_{3}} T_{3}^{1}(v) d v-\int_{\mathrm{R}_{4}} T_{4}^{1}(v) d v
$$

It is easy to check that $\phi T(v) d v$ depends neither on the decomposition of $T_{j}$ nor on the choice of $K_{j}$. 
We also call $T^{\prime}(v)\left(\right.$ resp. $v^{\alpha} T(v)$ ) the hyperfunction on $\mathbf{R}^{\prime \prime}$ whose restriction to $\mathbf{R}_{j}$ is $T_{j}^{\prime}(v)$ (resp. $v^{\alpha} T_{j}(v), \alpha \in N$ ). If $T(v)$ is without mass, $v^{\alpha} T(v)$ is too, for any integer $\alpha$.

LEMma 20. Let $T \in B\left(\mathbf{R}^{\prime \prime}\right)$ have mass $\Sigma_{\alpha \in \mathbb{N}} a_{\alpha} \delta^{(\alpha)}$; then

$$
\oint v^{\alpha} T(v) d v=(-1)^{\alpha} \alpha ! a_{\alpha} \quad(\alpha \in N) .
$$

Proof. We show first that if $T$ is without mass (that is $a_{\alpha}=0$ for every $\alpha)$, the shuttle integrals of all hyperfunctions $v^{\alpha} T(v)$ vanish; using the last remark above, it is enough to show that $\oint T(v) d v=0$. For computing this integral, we can choose $K_{j}, T_{j}^{1}, T_{j}^{2}$ such that

$$
\left.T_{1}^{1}\right|_{R^{+}}=\left.T_{2}^{1}\right|_{R^{+}},\left.\quad T_{1}^{1}\right|_{R^{-}}=\left.T_{4}^{1}\right|_{R^{-}},\left.\quad T_{3}^{1}\right|_{R^{-}}=\left.T_{2}^{1}\right|_{R^{-}},\left.\quad T_{3}^{1}\right|_{R^{+}}=\left.T_{4}^{1}\right|_{R^{+}}
$$

But then $T_{1}^{1}+T_{3}^{1}$ and $T_{2}^{1}+T_{4}^{1}$ coincide on $\mathrm{R}^{+}$and on $\mathrm{R}^{-}$, and also near the origin, since $T_{1}+T_{3}=T_{2}+T_{4}$ and the $T_{j}^{2}$ vanish there; there follows the equality $T_{1}^{1}+T_{3}^{1}=T_{2}^{1}+T_{4}^{1 \cdot}$, and thus $\oint T(v) d v=0$.

Now $T$ is always the sum of a hyperfunction without mass and the hyperfunction $\left(\Sigma a_{\alpha} \delta^{(\alpha)}, 0,0,0\right)$, and

$$
\oint v^{\alpha} T(v) d v=\int_{R_{1}} v^{\alpha}\left(\sum a_{\alpha} \delta^{(\alpha)}\right) d v=(-1)^{\alpha} \alpha ! a_{\alpha} \text {. Q.E.D. }
$$

REMARK 21. In particular a hyperfunction $T$ on $\mathbf{R}^{\prime \prime}$ is without mass if and only if the shuttle integrals of the $v^{\alpha} T(v)$ all vanish. Carrying this result on $\mathbf{R}^{2}-\{0\}$ by way of $Q^{\prime \prime-1}$, it is easy to see that a $G_{0}$-invariant hyperfunction $f(x, y)$ on $\mathrm{R}^{2}-\{0\}$ can be extended to a $G_{0}$-invariant hyperfunction on $\mathbf{R}^{2}$ if and only if the hyperfunctions on $\mathbf{R}^{+}$one gets by integrating $\left(x^{2}-y^{2}\right)(x y)^{\alpha} f(x, y)$ on the circles centered at the origin, vanish for every integer. If $f$ is a distribution which can be extended to a distribution on $\mathbf{R}^{2}$, one can get this result directly from Proposition 13 by integration by parts.

Noting that $\int_{\mathbf{R}} S^{\prime}(v) d v=0$ if $S(v)$ is a compactly supported hyperfunc-

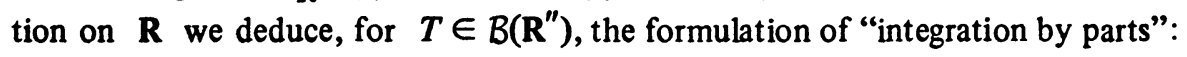

$$
\oint v^{\alpha} T^{\prime}(v) d v+\alpha \oint v^{\alpha-1} T(v) d v=0 \quad(\alpha \in \mathrm{N}) .
$$

LEMma 22. $T \in B\left(\mathbf{R}^{\prime \prime}\right)$ has a primitive in $B\left(\mathbf{R}^{\prime \prime}\right)$ if and only if its shuttle integral vanishes.

Proof. In a decomposition $T=T^{1}+T^{2}$ as above, $T^{2}$ always has a primitive $S^{2}$ in $B\left(R^{\prime \prime}\right)$ : one can choose $S_{j}^{2}$ to be the primitive of $T_{j}^{2}$ vanishing in a neighborhood of the origin. As the shuttle integral of $T^{2}$ vanishes, it is enough to prove the lemma for $T=T^{1}$. 
Let $S_{1}^{1}$ be a primitive of $T_{1}^{1}, S_{2}^{1}$ the primitive of $T_{2}^{1}$ which coincides with $S_{1}^{1}$ on $\mathrm{R}^{+}, S_{3}^{1}$ the primitive of $T_{3}^{1}$ which coincides with $S_{2}^{1}$ on $\mathrm{R}^{-}$, $S_{4}^{1}$ the primitive of $T_{4}^{1}$ which coincides with $S_{3}^{1}$ on $R^{+}$. Evidently $T$ will have a primitive in $B\left(R^{\prime \prime}\right)$ if and only if $S_{4}^{1}$ coincides with $S_{1}^{1}$ on $R^{-}$. But $S_{j}^{\prime}(v)$ is constant for $v$ big enough and for $v$ small enough, and the difference between these two constants is $\int_{\mathbf{R}_{j}} T_{j}^{1}(v) d v$. The conclusion follows immediately. Q.E.D.

COROLlary 23. A hyperfunction on $\mathbf{R}^{\prime \prime}$ is indefinitely integrable in $B\left(\mathbf{R}^{\prime \prime}\right)$ if and only if it is without mass.

Proof. Let $\Sigma a_{\alpha} \delta^{(\alpha)}$ be the mass of $T \in B\left(R^{\prime \prime}\right)$. According to Lemmas 20 and $22, T$ has a primitive $S \in B\left(\mathbf{R}^{\prime \prime}\right)$ if and only if $a_{0}=0$; furthermore

$$
\oint S(v) d v=-\oint v S^{\prime}(v) d v=-\oint v T(v) d v=a_{1} .
$$

The result follows by induction.

Proposition 24. Every linear differential equation with analytic coefficients is solvable in the space of hyperfunctions on $\mathbf{R}^{\prime \prime}$ without mass.

PROOF. One can solve any equation (E) with analytic coefficients in $B(R)$, and also extend any solution on some interval to a solution on a larger interval (Komatsu [3, Theorems 3.1 and 3.2]). Thus we can solve (E) in $B\left(R^{\prime \prime}\right)$ in the particular case where $T=\left(T_{4}, T_{3}, T_{3}, T_{4}\right)$ : it is enough to solve (E) on $\mathrm{R}^{+}$with second side $\left.T_{3}\right|_{\mathrm{R}^{+}}=T_{4} \mathrm{R}_{\mathrm{R}^{+}}$, and then extend the solution to a solution on $R$ with second side $T_{3}$, and with second side $T_{4}$ separately: the solution one gets in this way still has the form $\left(S_{4}, S_{3}, S_{3}, S_{4}\right)$, and thus is without mass.

So it is enough to show that (E) is solvable with second side $\left(T_{1}-T_{4}\right.$, $\left.T_{2}-T_{3}, 0,0\right)$, assuming that $\left(T_{1}, T_{2}, T_{3}, T_{4}\right)$ is without mass; that is to say $T_{1}-T_{4}=T_{2}-T_{3}$. But $T_{1}-T_{4}=T_{2}-T_{3}$ is supported in $\overline{\mathrm{R}^{+}}$. If $S_{1}$ is a solution of (E) in $B(R)$ for the second side $T_{1}-T_{4}=T_{2}-T_{3}$ which vanishes in $\mathbf{R}^{-}$(that is, which extends the trivial solution of the equation restricted to $\left.\mathbf{R}^{-}\right)$, the hyperfunction $\left(S_{1}, S_{1}, 0,0\right) \in B\left(R^{\prime \prime}\right)$ is clearly a solution of $(E)$ in $B\left(R^{\prime \prime}\right)$ in this case, and it is still without mass. Q.E.D.

REMARK. It is not true that any differential equation with analytic coefficients (on $\mathbf{R}$ or $\mathbf{R}^{\prime \prime}$, it is the same thing) is solvable in $B\left(\mathbf{R}^{\prime \prime}\right)$; as a matter of fact $\partial_{v}$ is not: see Lemma 22 .

3. Invariant equations. $P=P(I I)$ is a linear differential operator on $\mathbf{R}^{n}$, which is a polynomial (with complex coefficients) in an operator $\Pi$ with real 
constant coefficients, homogeneous of degree 1 or 2 . We assume that the degree of $P$ is at least 1 , and $n>1$. Let $G_{n}$ be the group of linear transformations of $\mathbf{R}^{n}$, of determinant $\pm 1, G$ the subgroup of $G_{n}$ which leaves $P$ invariant (that is, which leaves $\Pi$ invariant), and $G_{0}$ the connected component of the identity of $G$.

We recall that we want to answer the question: if $f \in B\left(R^{n}\right)$ is $G$ - (resp. $G_{0}$-) invariant, are there $G$ - (resp. $G_{0}$ ) invariant solutions $u \in B\left(\mathbf{R}^{n}\right)$ of the equation $P u=$ $f$, and how many?

(A) The case where $\Pi$ is of degree one. We do not lose anything by assuming $\Pi=\partial_{1}$. $G$ (resp. $G_{0}$ ) is then the group of linear transformations of determinant \pm 1 which do not move the first vector of the basis (resp. its connected component); and the orbits of $G$ (resp. $G_{0}$ ) are evidently

the whole of $\mathbf{R}^{n}$ minus the first axis $D_{1}$, as soon as $n>2$ (if $n=2$, $\mathbf{R}^{n}-D_{1}$ decomposes into orbits which are straight lines or pairs of straight lines parallel to $D_{1}$ ),

each point on $D_{1}$.

Put $\left(x_{1}, \cdots, x_{n}\right)=\left(x_{1}, x^{\prime}\right)$ with $x^{\prime} \in \mathrm{R}^{n-1}$. The group $G$ (resp. $\left.G_{0}\right)$ is the group of matrices $\left(\begin{array}{ll}1 & A \\ 0 & B\end{array}\right)$ where $B \in G_{n-1}$ (resp. $\operatorname{SL}(n-1, \mathbf{R})$ ), and $A \in \mathrm{R}^{n-1}$. A $G$ - (resp. $G_{0}$ ) invariant hyperfunction $f$ on $\mathbf{R}^{n}$ satisfies $f\left(x_{1}, x^{\prime}\right)=$ $f\left(x_{1}+A x^{\prime}, B x^{\prime}\right)$ and, by differentiating with respect to $A, \partial_{x_{1}} f \cdot x^{\prime}=0$. Thus $f$ is constant (with respect to $x_{1}$ ) on each connected component of $\mathrm{R}^{n}-D_{1}$.

Let $k$ be the constant term of $P$. If $k=0$, and if $u$ is an invariant solution of the equation $P u=f, u$ is constant with respect to $x_{1}$ on each connected component of $\mathbf{R}^{n}-D_{1}$, so that $P u=f$ vanishes there, that is, $f$ is supported on $D_{1}$. If $k \neq 0$ and if $f$ is equal to $C\left(x^{\prime}\right)$ on some connected component of $\mathbf{R}^{n}-D_{1}$, any invariant solution $u$ must be equal to $C\left(x^{\prime}\right) / k$ there, so that by substracting from $u$ the locally integrable function of $x_{1}$ which is equal to $C\left(x^{\prime}\right) / k$ in each connected component, one obtains a solution of the equation with second side $f$ minus the locally integrable function of $x_{1}$ which is equal to $C\left(x^{\prime}\right)$ in each connected component.

In both cases, one is led to solve the equation $P u=f$ with a second side $f$ supported on $D_{1}$. We distinguish two cases:

(1) If $n>2$, the invariance of $f$ under the subgroup of matrices $\left(\begin{array}{ll}1 & 0 \\ 0 & B\end{array}\right)$ implies that $f\left(x_{1}\right) \otimes \delta\left(x^{\prime}\right)$, since $\delta\left(x^{\prime}\right)$ is the only hyperfunction supported at the origin of $\mathbf{R}^{n-1}$ and invariant under $\operatorname{SL}(n-1, \mathbf{R})$. If $g\left(x_{1}\right)$ is a solution of $P\left(\partial_{1}\right) g\left(x_{1}\right)=f\left(x_{1}\right)$ then $u(x)=g\left(x_{1}\right) \otimes \delta\left(x^{\prime}\right)$ is obviously a $G$-invariant solution of $P u=f$.

(2) If $n=2$, the second side can be written

$$
\sum_{\alpha \in \mathbb{N}} f_{\alpha}\left(x_{1}\right) \otimes \delta^{(\alpha)}\left(x_{2}\right)
$$


where $f_{\alpha} \in B(\mathbf{R})$ must vanish for odd $\alpha$ if $f$ is $G$-invariant, and

$$
u(x)=\sum_{\alpha \in \mathbb{N}} g_{\alpha}\left(x_{1}\right) \otimes \delta^{(\alpha)}\left(x_{2}\right)
$$

where $g_{\alpha}$ is a solution of $P\left(\partial_{1}\right) g_{\alpha}\left(x_{1}\right)=f_{\alpha}\left(x_{1}\right)$ is again a $G$ - or $G_{0}$-invariant solution, at least formally. Now if the series (*) converges in $B\left(R^{2}\right)$, one can write

$$
f_{\alpha}\left(x_{1}\right)=a_{\alpha}\left[F_{\alpha}\left(z_{1}\right)\right]_{z_{1}=x_{1}} \text { where } \limsup _{\alpha \rightarrow \infty}\left(\left|a_{\alpha}\right| \alpha !\right)^{1 / \alpha}=0,
$$

and the $F_{\alpha}$ are holomorphic functions on $\mathbf{C}-\mathbf{R}$, uniformly bounded on every compact set. But then there are solutions $G_{\alpha}\left(z_{1}\right)$ of the equations $P\left(\partial_{z_{1}}\right) G_{\alpha}\left(z_{1}\right)=F_{\alpha}\left(z_{1}\right)$ which have the same property (by the Banach homomorphism theorem, and since $\mathbf{C}-\mathbf{R}$ is simply connected), and choosing $g_{\alpha}\left(x_{1}\right)=a_{\alpha}\left[G_{\alpha}\left(z_{1}\right)\right]_{z_{1}=x_{1}}$ leads to a series (**) which is evidently convergent in $B$.

We summarize the discussion above in the following statement:

Proposition 25. Let $k$ be the constant term of $P$, and $\Pi$ be of degree 1. If $f \in B\left(\mathbf{R}^{n}\right)$ is $G$ - (resp. $G_{0^{-}}$) invariant, the equation $P(I I) u=f$ always has $G$ - (resp. $G_{0^{-}}$) invariant solutions if $k \neq 0$. If $k=0$, it has invariant solutions if and only if $f$ is supported on the G-invariant straight line of $\mathbf{R}^{n}$.

REMARK. The example in Rais [6] is covered by the second part of this proposition: one takes $P(\Pi)=\partial_{1}$ in $R^{2}$, and $G_{0}$ is the group of transvections $\left(\begin{array}{ll}1 & a \\ 0 & 1\end{array}\right)(a \in \mathbf{R})$. The equation $\partial_{1} u=1$ has no invariant solution, as 1 is not supported on the first axis.

(B) The case where $\Pi$ is of degree two: First considerations. We do not lose anything by assuming that $\Pi$ is diagonal:

$$
\Pi=\partial_{1}^{2}+\cdots+\partial_{p}^{2}-\partial_{p+1}^{2}-\cdots-\partial_{p+q}^{2} \quad(p+q \leqslant n) .
$$

Then $G$ (resp. $\left.G_{0}\right)$ is the group of matrices $\left(\begin{array}{ll}A & B \\ 0 & C\end{array}\right)$ where $B$ is $(p+q) \times$ $(n-p-q), A$ is in the orthogonal group $O(p, q)$ (resp. its connected component), and $C$ is in the group $G_{n-p-q}$ of matrices of $\mathrm{GL}(n-p-q, \mathbf{R})$ with determinant \pm 1 (resp. in $\operatorname{SL}(n-p-q, \mathbf{R})$ ). The orbits of $G$ (resp. $\left.G_{0}\right)$ are the orbits of $O(p, q)$ (resp. its connected component) in $\mathbf{R}^{p+q} \times\{0, \cdots, 0\}$, the complement of $\mathbf{R}^{p+q} \times\{0, \cdots, 0\}$ in $\mathbf{R}^{n}$ is composed of 0,1 , or $\infty$ orbits according to whether $n-p-q$ is zero, larger than one, or equal to one.

Using, as in the first case, the invariance of a hyperfunction under the subgroups $\left(\begin{array}{ll}1 & 0 \\ 0 & C\end{array}\right)$ and $\left(\begin{array}{ll}A & B \\ 0 & 1\end{array}\right)$, one sees that the invariant hyperfunctions on $R^{n}$ are the sums of 
a constant (or pair of constants) with respect at least to $\left(x_{1}, \cdots, x_{p+q}\right)$, one which can be written $\Sigma f_{\alpha}\left(x_{1}, \cdots, x_{p+q}\right) \otimes \delta^{(\alpha)}\left(x_{p+q+1}, \cdots, x_{n}\right)$ where $f$ is a hyperfunction on $\mathrm{R}^{p+q}$ invariant under $O(p, q)$ (resp. its connected component), and $\delta^{(\alpha)}$ is invariant under $G_{n-p-q}($ resp. SL $(n-p-q, \mathbf{R})$ ).

If $p+q<n$, reasoning as in (A), we see that if $k=0$ ( $k$ is, as above, the constant term of the polynomial $P$ ), the equation $P u=f$ will have invariant solutions only if $f$ is supported in $\mathbf{R}^{p+q} \times\{0, \cdots, 0\}$; if that is so, on if $k \neq 0$, we are led as above to solve the equation $P u=f$ in the space of hyperfunctions on $\mathbf{R}^{p+q}$ invariant under $O(p, q)$ (resp. its connected component).

It is thus enough to deal with the case $p+q=n$, in other words the case where $\Pi$ is of principal type. This is done in the following paragraph. However we will deal here with an easy particular case: that where $\Pi$ is elliptic.

This happens if and only if $p q=0$, and $G=O(n)$ and $G_{0}$ are then compact. The equation $P u=f$, for any second side $f \in B\left(R^{n}\right)$, is then solvable since $P$ is elliptic (see [2]), and if $u$ is a solution,

$$
\tilde{u}(x)=\int_{G \text { or } G_{0}} u(g x) d g,
$$

where $d g$ stands for the normalized Haar measure of $G$ or $G_{0}$, is obviously an invariant solution if $f$ is invariant.

Thus every invariant equation has an invariant solution in this case.

(C) The interesting case. $P=P(I)$ is now a differential operator which is a nonconstant polynomial with complex coefficients of a linear differential operator $\Pi$ with constant real coefficients, homogeneous of degree 2 , of principal type, nonelliptic.

We do not lose anything by assuming that $\Pi$ is diagonal:

$$
\Pi=\partial_{1}^{2}+\cdots+\partial_{p}^{2}-\partial_{p+1}^{2}-\cdots-\partial_{p+q}^{2} \quad(p+q=n, p q \neq 0) .
$$

The group $G$ is self-transposed, and it is the group $O(p, q)$ of matrices which leave invariant the quadratic form

$$
Q(x)=x_{1}^{2}+\cdots+x_{p}^{2}-x_{p+1}^{2}-\cdots-x_{p+q}^{2} .
$$

By Propositions 5 and 7 and Remark 6 if $p q>1$, and by Propositions 16 and 17 if $p q=1$, we can transform the equation $P u=f$ restricted to $R^{p+q}-\{0\}$ into a differential equation on $\mathbf{R}, \mathbf{R}^{\prime}$ or $\mathbf{R}^{\prime \prime}$ : if $f=Q^{-1}(T)$ and $u=Q^{-1}(S)$ (or replacing $Q$ by $Q^{\prime}$ or $Q^{\prime \prime}$ ), $S$ and $T$ being hyperfunctions on $\mathbf{R}, \mathbf{R}^{\prime}$ or $\mathbf{R}^{\prime \prime}$, this equation is equivalent to the differential equation (on $\mathbf{R}, \mathbf{R}^{\prime}$ or $\mathbf{R}^{\prime \prime}$ ) obtained by transforming $P$ under one of the diffeomorphisms $\theta_{k l}$ of the proof of Proposition 5: 


$$
P\left(4 v \partial_{v}^{2}+2(p+q) \partial_{v}\right) S(v)=T(v) .
$$

It is known (Komatsu [3, Theorem 3.1]) that equation (E) is solvable in $B(R)$ for any second side in $B(R)$; this is still true in $B\left(R^{\prime}\right)$ : first one solves (E) on the negative open half-line, then one extends the solution separately to a solution on $\mathbf{R}_{1}$ and to a solution on $\mathbf{R}_{\mathbf{2}}$, and this is always possible (Komatsu [3, Theorem 3.2]). But it is not true in $B\left(\mathbf{R}^{\prime \prime}\right)$ (cf. the remark at the end of §2).

However if $\left.f\right|_{R^{2}-\{0\}}=Q^{n-1}(T)$ has a $G_{0}$-invariant extension on $\mathbf{R}^{2}$ (which is certainly the case if $f \in B\left(R^{2}\right)$ is $G_{0}$-invariant!), the mass of $T$ is zero by Theorem 18, and Proposition 24 then states that equation $(E)$ has a solution $S \in B\left(\mathbf{R}^{\prime \prime}\right)$ with mass zero. We have thus $P u=f$ in $\mathbf{R}^{2}-\{0\}$ with $u=Q^{\prime \prime-1}(T) \in B\left(\mathbf{R}^{2}-\{0\}\right)$ having a $G_{0}$-invariant extension on $\mathbf{R}^{2}$ by Theorem 18.

If on the other hand $p q>1$, solving equation $(E)$ in $B(R)$ or $B\left(R^{\prime}\right)$ is the same as solving the equation $P u=f$ in the space of hyperfunctions on $\mathbf{R}^{p+q}-\{0\}$ invariant under $G$ or $G_{0}$, and we know (Theorem 10 or Corollaries 11,19$)$ that any such solution has a $G_{0}$-invariant extension at the origin, thus easily a $G$-invariant one by symmetrisation, if $f$ itself is $G$-invariant.

In short, and in any case, the equation $P u=f$, where $f \in B\left(R^{p+q}\right)$ is invariant under $G$ (resp. $\left.G_{0}\right)$, always has a solution $u \in B\left(R^{p+q}-\{0\}\right)$ on $\mathrm{R}^{p+q}-\{0\}$, invariant under $G\left(\right.$ resp. $\left.G_{0}\right)$, and admitting an extension $\tilde{u} \in B\left(R^{p+q}\right)$ at the origin, invariant under $G$ (resp. $\left.G_{0}\right)$.

But then $P \widetilde{u}=f+g$ where $g$ is at least $G_{0}$-invariant, and supported at the origin. By Proposition 8 if $p q>1$ and Proposition 15 if $p q=1, g$ can be written $g=\Sigma_{\alpha \in \mathrm{N}} a_{\alpha} \Pi^{\alpha} \delta$. Let $E$ be the $G$-invariant fundamental solution of $P$ given by the theorem of Atiyah-Rais cited in the introduction. The series $v=$ $\Sigma_{\alpha \in \mathrm{N}} a_{\alpha} \Pi^{\alpha} E$ is convergent in $B$ : as it would be too long here to give a direct proof of this, as in Lemma 14, we define $v$ as the convolution product of $E$ and $g$, which exists as $g$ is compactly supported (see $[10$, p. 61$])$. We have then

$$
P(\tilde{u}-v)=f+g-P(E * g)=f .
$$

Moreover as $E$ and $g$ are $G$-invariant, so is $v$, and $\tilde{u}-v$ is a $G$ - (resp. $G_{0}-$ ) invariant solution of the equation on the whole space $R^{p+q}$.

Finally we have proved:

THEOREM 26. Let $G$ be the subgroup of $\mathrm{GL}(p+q, \mathrm{R})$ leaving invariant the operator $\Pi=\partial_{1}^{2}+\cdots+\partial_{p}^{2}-\partial_{p+1}^{2}-\cdots-\partial_{p+q}^{2}, G_{0}$ its connected component of the identity, and $P$ a polynomial with complex coefficients. For any hyperfunction $f$ on $\mathrm{R}^{p+q}$ invariant under $G$ (resp. $G_{0}$ ), there exists a hyperfunction solution $u$ on $\mathrm{R}^{p+q}$ invariant under $G$ (resp. $G_{0}$ ) of the equation $P(I I) u=f$. 
COROllary 27. Let $\square$ stand for the D'Alembert operator of $\mathbf{R}^{p+1}$ and $k$ be a complex number. The equation $(\square+k) u=f$ has a hyperfunction solution $u$ on $\mathrm{R}^{p+1}$ invariant under the Lorentz group, for any hyperfunction $f$ on $\mathbf{R}^{p+1}$ invariant under the Lorentz group.

Proof. $\square+k$ is the operator of Theorem 26, where $q=1$ and $P$ is of degree 1 , and $G_{0}$ is then a subgroup of the Lorentz group with finite index (in fact 2). If $\bar{u}$ is a $G_{0}$-invariant solution given by Theorem 26 ,

$$
u\left(x_{1}, x_{2}, \cdots, x_{p+1}\right)=1 / 2\left[\bar{u}\left(x_{1}, x_{2}, \cdots, x_{p+1}\right)+\bar{u}\left(x_{2}, x_{1}, \cdots, x_{p+1}\right)\right]
$$

is a solution invariant under the Lorentz group.

Finally we gather the above results on invariant equations in the following statement:

THEOREM 28. Let $\Pi$ be a linear differential operator on $\mathbf{R}^{n}$, with constant real coefficients, homogeneous of degree 1 or 2 . Let $\Delta$ be the subspace of $\mathbf{R}^{n}$ generated by vector $\Pi$ if $\Pi$ is of degree 1, by the partial derivatives of $\Pi$ if $\Pi$ is of degree 2. Let $P$ be a polynomial with complex coefficients, $k$ its constant term, $G$ the group of linear transformations of $\mathbf{R}^{n}$ with determinant \pm 1 and leaving $\Pi$ invariant, $G_{0}$ its connected component of the identity, and $f$ a hyperfunction on $\mathbf{R}^{n}$ invariant under $G$ (resp. $G_{0}$ ).

If $k \neq 0$, the equation $P(I I) u=f$ has a hyperfunction solution $u$ on $\mathbf{R}^{n}$ invariant under $G$ (resp. $\left.G_{0}\right)$.

If $k=0$, the equation $P(\Pi) u=f$ has a hyperfunction solution $u$ on $\mathbf{R}^{n}$ invariant under $G$ (resp. $G_{0}$ ) if and only if $f$ is supported on $\Delta$.

Proof. If $\Pi$ is of degree 1 , this is the contents of Proposition 25. If II is of degree 2, it follows easily from Theorem 26 and from the considerations of $\S 3 \mathrm{~B}$.

(D) The homogeneous equation. We still have to answer the question: how many invariant solutions are there; that is to say, what is the dimension of the space of invariant solutions of the homogeneous equation?

We study here only the case where $\Pi$ is of degree 2 and of principal type. Conclusion in the other cases would follow easily from the present study and the considerations of $\S 3 \mathrm{~A}$ and $\S 3 \mathrm{~B}$. Finally, the case where $\Pi$ is elliptic is classical and we do not treat it.

Let $B^{G_{0}}$ be the sheaf on $\mathrm{R}^{p+q}$ of locally $G_{0}$-invariant hyperfunctions: according to Lemma 1 , and with the notations of $\S 1$, it is the kernel of the sheaf morphism 


$$
B \stackrel{X_{i j}, Y_{i j}}{\longrightarrow} B^{(p+q)(p+q-1) / 2} \text {. }
$$

We also write $B^{G_{0}, P}$ for the subsheaf of $B^{G_{0}}$ of the $G_{0}$-invariant solutions $u$ of the homogeneous equation $P(\Pi) u=0$, and we use analogous notations with $G_{0}$ replaced by $G$; but the presheaf $B^{G}$ of locally $G$-invariant hyperfunctions is no more a sheaf.

Lemma 29. If $p q>1, B^{G_{0}, P}\left(\mathbf{R}^{p+q}-\{0\}\right) / B^{G_{0}, P}\left(\mathbf{R}^{p+q}\right)$ is canonically isomorphic to

$$
B_{\{0\}}^{G_{0}}\left(\mathrm{R}^{p+q}\right) / P(\mathrm{II}) B_{\{0\}}^{G_{0}}\left(\mathrm{R}^{p+q}\right) .
$$

In any case $B^{G, P}\left(\mathbf{R}^{p+q}-\{0\}\right) / B^{G, P}\left(\mathbf{R}^{p+q}\right)$ is canonically isomorphic to

$$
B_{\{0\}}^{G}\left(\mathbf{R}^{p+q}\right) / P(\Pi) B_{\{0\}}^{G}\left(\mathbf{R}^{p+q}\right) .
$$

Proof. Since $P(I I)$ is surjective in $B^{G_{0}}\left(\mathbf{R}^{p+q}\right)$ (Theorem 26), and since every $G_{0}$-invariant hyperfunction on $R^{p+q}-\{0\}$ is the restriction of some $G_{0}$-invariant hyperfunction on $\mathbf{R}^{p+q}$, as $p q>1$ (Theorem 10), Remark 3 applies with $F=B^{G} 0, P=P(I I), M=\mathbf{R}^{p+q}, U=\mathbf{R}^{p+q}-\{0\}, F=\{0\}$, and the first statement follows. For the second statement, it is enough to remark that, although $B^{G}$ is not a sheaf, we have still an exact commutative diagram analogous to that of Lemma 2: as $P(\Pi)$ is again surjective in $B^{G}\left(R^{p+q}\right)$ (Corollary 27), and the $G$-invariant hyperfunctions on $\mathbf{R}^{p+q}-\{0\}$ have $G$-invariant extensions at the origin (Corollaries 11 and 19), the result is the same. Q.E.D.

Let $m$ be the degree of $P$.

LEMmA 30. The space $B_{\{0\}}^{G}$ of $G_{0}$-invariant hyperfunctions supported at the origin contains only G-invariant hyperfunctions. The dimension of the quotient

$$
B_{\{0\}}^{G} / P(\Pi) B_{\{0\}}^{G}=B_{\{0\}}^{G_{0}} / P(\Pi) B_{\{0\}}^{G_{0}}
$$

is equal to $\mathrm{m}$.

Proof. The first statement is contained in Propositions 8 and 15, which also show that $B_{\{0\}}^{G}$ is the space of the series $\Sigma_{\alpha \in N} a_{\alpha} \Pi^{\alpha} \delta$ such that

$$
\limsup _{\alpha \rightarrow \infty}\left(\left|a_{\alpha}\right|(2 \alpha) !\right)^{1 / 2 \alpha}=0 .
$$

Thus we have to compute the codimension of the range of multiplication by the polynomial $P(X)$ in the space of the series $\Sigma a_{\alpha} X^{\alpha}$ whose coefficients satisfy ( $\left.C^{\prime}\right)$. But $\left(C^{\prime}\right)$ means that $\Sigma a_{\alpha}(2 \alpha) ! / z^{2 \alpha+1}$ is holomorphic outside the origin; 
it is a classical result that this is equivalent to saying that $\Sigma a_{\alpha} z^{2 \alpha}$ is an entire function of zero exponential type. Given such an entire function, one can always find, and in a unique way, a polynomial $Q(X)$ of degree at most $m-1$ and an entire function $\Sigma b_{\alpha} z^{2 \alpha}$ such that

$$
\sum a_{\alpha} X^{\alpha}=P(X)\left(\sum b_{\alpha} X^{\alpha}\right)+Q(X) .
$$

As $Q\left(z^{2}\right)$ and $\Sigma a_{\alpha} z^{2 \alpha}$ are of zero exponential type, so is $P\left(z^{2}\right)\left(\Sigma b_{\alpha} z^{2 \alpha}\right)$, and so is $\Sigma b_{\alpha} z^{2 \alpha}$ itself, as $\left|P\left(z^{2}\right)\right|$ is bounded below outside some compact set.

In other words the coefficients $b_{\alpha}$ satisfy $\left(C^{\prime}\right)$, and the polynomials of degree at most $m-1$ are thus a complement of $P(\Pi) B_{\{0\}}^{G}$ in $B_{\{0\}}^{G}$. Q.E.D.

LEMma 31. The dimension of $B^{G, P}\left(\mathbf{R}^{p+q}-\{0\}\right)$ is $3 m$. The dimension of $B^{G_{0}, P}\left(\mathrm{R}^{p+q}-\{0\}\right)$ is $3 m$ if $p>1$ and $q>1,4 m$ if $p>1$ and $q=1$.

Proof. $B^{G, P}\left(\mathrm{R}^{p+q}-\{0\}\right)$ is the inverse image under $Q$ of the kernel in $B(\mathbf{R})$ of the operator

$$
P\left(4 v \partial_{v}^{2}+2(p+q) \partial_{v}\right) .
$$

This operator is of degree $2 m$ and has a singular point at the origin: its principal coefficient vanishes there with order $m$. It is then a consequence of Theorem 3.3 of Komatsu [3] that the dimension of its kernel in $B(\mathbf{R})$ is $3 m$.

If $p>1$ and $q>1, B^{G}=B^{G}$ (Remark 6).

If $p>1$ and $q=1, B^{G_{0}, P}\left(R^{p+1}-\{0\}\right)$ is the inverse image under $Q^{\prime}$ of the kernel of operator (*) in $B\left(R^{\prime}\right)$. As $(*)$ is regular on $R^{-}$, its kernel in $B\left(\mathbf{R}^{-}\right)$has dimension $2 m$; thus its kernel in $B(\mathbf{R})$ contains $m$ linearly independent hyperfunctions supported in $\overline{R^{+}}$, and there follows that its kernel in $B\left(\mathbf{R}^{\prime}\right)$ has dimension $2 m+m+m=4 m$. Q.E.D.

THEOREM 32. The dimension of the space of G-invariant hyperfunctions $u$ on $\mathbf{R}^{p+q}$ which are solutions of the homogeneous equation $P(\Pi) u=0$ is $2 m$, where $m$ is the degree of $P$. The dimension of the space of those which are only $G_{0}$-invariant is $2 m$ if $p>1$ and $q>1,3 m$ if $p>1$ and $q=1$.

Proof. As $P(I)$ has constant coefficients, it is one-to-one in the space of hyperfunctions supported at the origin. So the canonical restrictions

$$
\begin{aligned}
B^{G_{0}, P}\left(\mathbf{R}^{p+q}\right) & \rightarrow B^{G_{0}, P}\left(\mathbf{R}^{p+q}-\{0\}\right), \\
B^{G, P}\left(\mathbf{R}^{p+q}\right) & \rightarrow B^{G, P}\left(\mathbf{R}^{p+q}-\{0\}\right)
\end{aligned}
$$

are injective. The theorem follows then from Lemmas 29,30 and 31. 
REMARK. We find here as a corollary the result of Methee [4]: there are 3 independent solutions of the equation $(\square+k) u=0$, where $\square$ is the D'Alembert operator, which are invariant under the connected component of the Lorentz group, and only 2 invariant under the whole Lorentz group.

There remains only the computing of the dimension of the space of $G_{0}$-invariant solutions of the homogeneous equation in case $p=q=1$. But in this case, we still have an exact commutative diagram, analogous to that of Lemma 2:

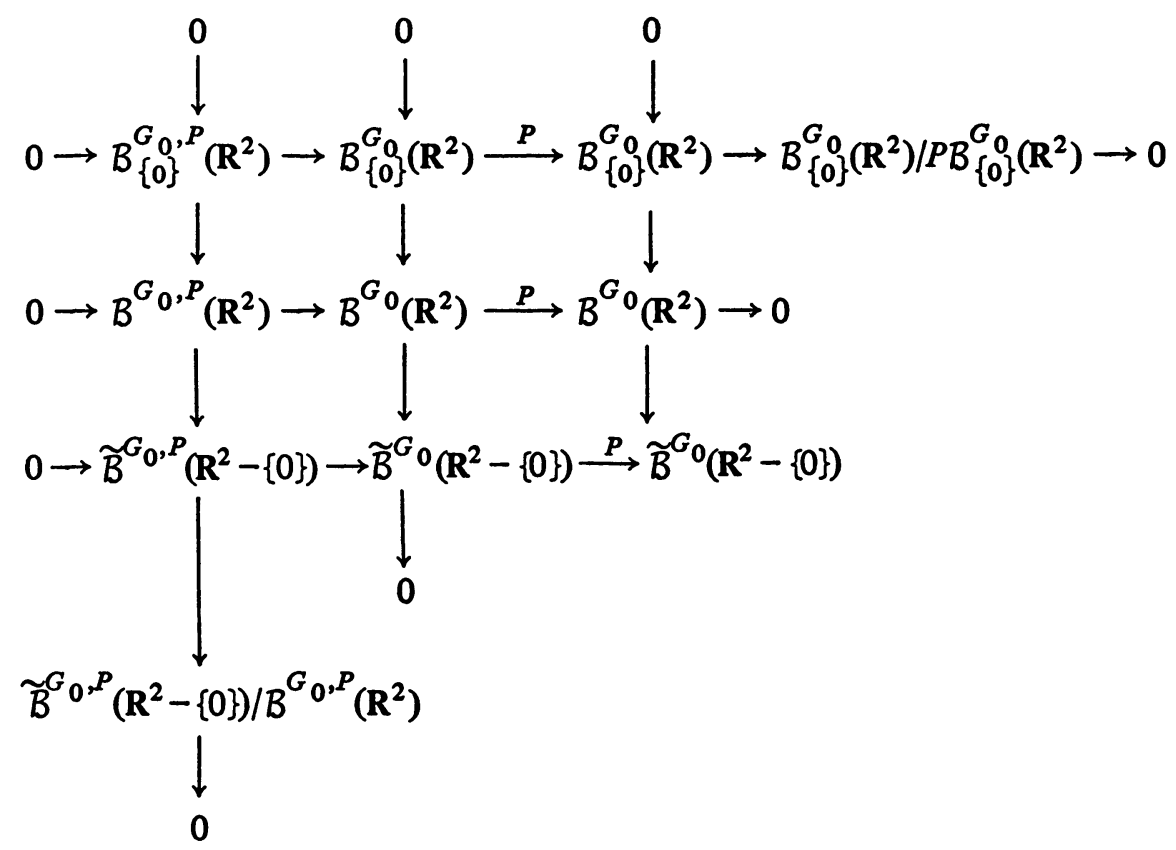

in view of Theorem 26 , where $\widetilde{B}^{G_{0}}\left(\mathbf{R}^{2}-\{0\}\right)$ is the space of $G_{0}$-invariant hyperfunctions on $\mathbf{R}^{2}-\{0\}$ which have a $G_{0}$-invariant extension at the origin. So $\widetilde{B}^{G_{0}, P}\left(\mathbf{R}^{2}-\{0\}\right) / B^{G_{0}, P}\left(\mathbf{R}^{2}\right)$ is isomorphic to $B_{\{0\}}^{G_{0}}\left(\mathbf{R}^{2}\right) / P B_{\{0\}}^{G_{0}}\left(\mathbf{R}^{2}\right)$, and so has dimension $m$ after Lemma 30 . The canonical restriction

$$
B^{G_{0}, P}\left(\mathbf{R}^{2}\right) \rightarrow \widetilde{B}^{G_{0}, P}\left(\mathbf{R}^{2}-\{0\}\right)
$$

is still one-to-one, and it is thus enough to determine the dimension of $\widetilde{B}^{G_{0}, P}\left(\mathbf{R}^{2}-\{0\}\right)$, which is the inverse image under $Q^{n}$ of the kernel of the operator (*) in the space of hyperfunctions on $\mathbf{R}^{\prime \prime}$ without mass.

THEOREM 33. The dimension of the space of $G_{0}$-invariant hyperfunctions $u$ on $\mathbf{R}^{2}$ which are solutions of the homogeneous equation $P(I I) u=0$ is $4 m$.

Proof. In view of the remarks above, it is obviously enough to show that the 
dimension of the kernel of (*) in the space of hyperfunctions on $\mathbf{R}^{\prime \prime}$ without mass is $5 m$.

A solution of the homogeneous equation on $\mathbf{R}_{1}^{+}=\mathbf{R}_{2}^{+}$depends on $2 m$ parameters, and there are $m$ more parameters as one extends to $R_{1}$, and $m$ more as one extends to $R_{2}$; choosing these $4 m$ parameters determines the solution everywhere except on $\overline{\mathbf{R}_{3}^{+}}$and on $\overline{\mathbf{R}_{4}^{+}}$. By adding $m$ more parameters, one can determine it on $\mathbf{R}_{3}$, and it is then determined everywhere except at the origin of $\mathbf{R}_{\mathbf{4}}$, and can clearly be extended to the whole of $\mathbf{R}^{\prime \prime}$. But it is easy to check that (*) is injective in the space of hyperfunctions supported at the origin. Thus $5 m$ is the dimension of the kernel of the operator (*), that is to say $P\left(4 v \partial_{v}^{2}+4 \partial_{v}\right)$, in $B\left(\mathbf{R}^{\prime \prime}\right)$, and we have left to show that this kernel contains only hyperfunctions without mass.

(a) If the constant term of $P$, say $k$, is not zero, and if $S(v)$ is a hyperfunction on $\mathbf{R}^{\prime \prime}$ such that $P\left(4 v \partial_{v}^{2}+4 \partial_{v}\right) S(v)=0$, we get for any integer $\alpha$, integrating by parts on $\mathbf{R}^{\prime \prime}$ and developing the polynomial $P$ :

$$
\begin{aligned}
0 & =\oint v^{\alpha} P\left(4 v \partial_{v}^{2}+4 \partial_{v}\right) S(v) d v=\sum_{j=1}^{\inf (m, \alpha)} A_{j} \oint v^{\alpha-j} S(v) d v+k \oint v^{\alpha} S(v) d v \\
& =\sum_{j=1}^{\inf (m, \alpha)} A_{j}(-1)^{\alpha-j}(\alpha-j) ! a_{\alpha-j}+(-1)^{\alpha} k \alpha ! a_{\alpha}
\end{aligned}
$$

by Lemma 20, where $\Sigma_{\alpha \in N} a_{\alpha} \delta^{(\alpha)}$ is the mass of $S$. As $k \neq 0$, these equations imply by induction that all $a_{\alpha}$ vanish.

(b) Moreover, if $k \neq 0$, any hyperfunction $S$ on $\mathbf{R}^{n}$ satisfying $P\left(4 v \partial_{v}^{2}+4 \partial_{v}\right) S(v)=T(v)$, with $T$ without mass, must be without mass, since there is at least one such (Proposition 24) and two of them differ from a hyperfunction without mass by (a).

(c) If $S \in B\left(\mathbf{R}^{\prime \prime}\right)$ satisfies $\left(4 v \partial_{v}^{2}+4 \partial_{v}\right)^{r} S(v)=T(v)$, where $r$ is some integer, and $T$ is without mass, $S$ also is without mass: in fact one shows exactly as in (b) that it is enough to prove it for $T=0$. Now this is obvious if $r=0$; if $r>0$, the equation

$$
\left(4 v \partial_{v}^{2}+4 \partial_{v}\right)\left(4 v \partial_{v}^{2}+4 \partial_{v}\right)^{r-1} S(v)=0
$$

means that $\left(4 v \partial_{v}^{2}+4 \partial_{v}\right)^{r-1} S(v)$ is a solution of the homogeneous equation $\left(4 v \partial_{v}^{2}+4 \partial_{v}\right) R(v)=0$. But the solutions of the last equation are the linear combinations of $1, \ln |v|$ and $H(v)$, where $H$ is the Heaviside function; since they are locally integrable, they all are without mass. Whence the conclusion by induction. 
(d) Since every polynomial is the product of some monomial by a polynomial with nonvanishing constant term, the theorem follows from (b) and (c). Q.E.D.

REMARK. The proof shows in fact that

$$
\widetilde{B}^{G_{0}, P}\left(\mathbf{R}^{2}-\{0\}\right)=B^{G_{0}, P}\left(\mathbf{R}^{2}-\{0\}\right) ;
$$

that is, every $G_{0}$-invariant solution of the homogeneous equation on $\mathbf{R}^{2}-\{0\}$ can be extended to a $G_{0}$-invariant solution on $\mathbf{R}^{2}$.

\section{REFERENCES}

1. M. F. Atiyah, Resolution of singularities and division of distributions, Comm. Pure Appl. Math. 23 (1970), 145-150. MR 41 \#815.

2. R. Harvey, Hyperfunctions and partial differential equations, Proc. Nat. Acad. Sci. U.S.A. 55 (1966), 1042-1046. MR 34 \#495.

3. H. Komatsu, An introduction to the theory of hyperfunctions, Hyperfunctions and Pseudo-Differential Equations, Lecture Notes in Math., vol 287, Springer-Verlag, Berlin and New York, 1973.

4. P. D. Methée, Sur les distributions invariantes dans le groupe des rotations de Lorentz, Comment. Math. Helv. 28 (1954), 225-269. MR 16, 255.

5. T. Miwa, On the existence of hyperfunctions solutions of linear differential equations of the first order with degenerate real principal symbols, Proc. Japan Acad. 49 (1973), 88-93.

6. M. Rais, Solutions élémentaires des opérateurs bi-invariants sur un groupe de Lie nilpotent, C. R. Acad. Sci. Paris Sér. A-B 273 (1971), A495-A498. MR 44 \#6908.

7. M. Riesz, L'integrale de Riemann-Liouville et le problème de Cauchy, Acta Math. 81 (1949), 1-223. MR 10, 713.

8. M. Sato, Theory of hyperfunctions. I, II, J. Fac. Sci. Univ. Tokyo 8 (1959), 139-193, 387-437. MR 22 \#4951; 24 \#A2237.

9. M. Sato, T. Kawai and M. Kasiwara, Microfunctions and pseudo-differential equations, Hyperfunctions and Pseudo-Differential Equations, Lecture Notes in Math., vol. 287, SpringerVerlag, Berlin and New York, 1973, pp. 265-529.

10. P. Shapira, Théorie des hyperfonctions, Lecture Notes in Math., vol. 126, SpringerVerlag, Berlin and New York, 1970.

11. G. de Rham, Sur la division de formes et de courants par une forme linéaire, Comment. Math. Helv. 28 (1954), 346-352. MR 16, 402.

12. P. D. Methée, Transformées de Fourier de distributions invariantes. II, C. R. Acad. Sci. Paris 241 (1955), 684-686. MR 17, 845.

13. G. de Rham, Solution élémentaire d'opérateurs différentiels du second ordre, Ann. Inst. Fourier (Grenoble) 8 (1958), 337-366. MR 22 \#8216.

14. L. Gårding and J. L. Lions, Functional analysis, Nuovo Cimento (10) 14 (1959), supplemento, 9-66. MR 22 \#8321.

15. A. Tengstrand, Distributions invariant under an orthogonal group of arbitrary signature, Math. Scand. 8 (1960), 201-218. MR 23 \#A3450.

16. M. Raïs, Les solutions invariantes de l'équation des ondes, C. R. Acad. Sci. Paris 259 (1964), 2169-2170.

17. V. Edén, Distributions invariant under the group of complex orthogonal transformations, Math. Scand. 14 (1964), 75-89. MR 30 \#4151.

IMSP, UNIVERSITE DE NICE, PARC VALROSE, 06 NICE, FRANCE 